\title{
Pion production and charged-particle multiplicity selection in relativistic nuclear collisions
}

\author{
K. L. Wolf, ${ }^{(a)}$ H. H. Gutbrod, ${ }^{\dagger(b)}$ W. G. Meyer, ${ }^{\dagger(c)}$ A. M. Poskanzer, ${ }^{*}$ A. Sandoval, ${ }^{\dagger(b)}$ R. Stock, ${ }^{\dagger(b)}$ \\ J. Gosset, ${ }^{*(d)}$ J.-C. Jourdain, ${ }^{*(\mathrm{e})}$ C. H. King, ${ }^{*(\mathrm{f})}$ G. King, ${ }^{*(\mathrm{~g})}$ Nguyen Van Sen, ${ }^{*(\mathrm{~h})}$ and G. D. Westfall*(i) \\ *Lawrence Berkeley Laboratory, Berkeley, California 94720 \\ and $^{\dagger}$ Gesellschaft für Schwerionenforschung, Darmstadt, Federal Republic of Germany
}

(Received 13 April 1982)

\begin{abstract}
Spectra of positive pions with energies of 15-95 MeV were measured for high energy proton, ${ }^{4} \mathrm{He}$, ${ }^{20} \mathrm{Ne}$, and ${ }^{40} \mathrm{Ar}$ bombardments of targets of ${ }^{27} \mathrm{Al},{ }^{40} \mathrm{Ca},{ }^{107,109} \mathrm{Ag},{ }^{197} \mathrm{Au}$, and ${ }^{238} \mathrm{U}$. A Si-Ge telescope was used to identify charged pions by $d E / d x-E$ and, in addition, stopped $\pi^{+}$were tagged by the subsequent muon decay. In all, results for 14 targetprojectile combinations are presented to study the dependence of pion emission patterns on the bombarding energy (from $E / A=0.25$ to $2.1 \mathrm{GeV}$ ) and on the target and the projectile masses. In addition, associated charged-particle multiplicities were measured in an 80paddle array of plastic scintillators, and used to make impact parameter selections on the pion-inclusive data.
\end{abstract}

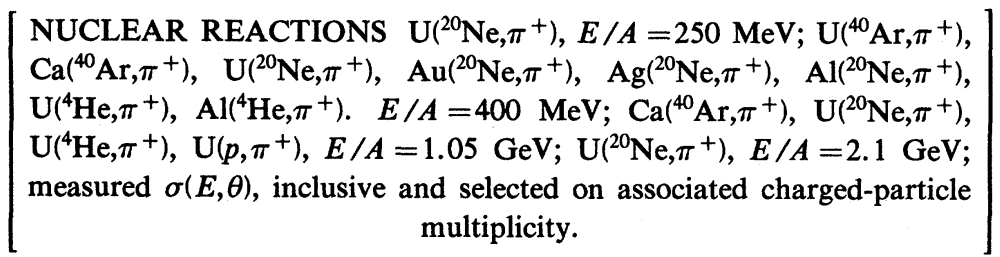

\section{INTRODUCTION}

Pion production cross sections for $p+p$ collisions have provided some of the basic data in the study of pion physics, and further understanding has been gained with proton- and neutron-induced pion production on heavier nuclei. Also for heavy ion collisions at relativistic or near-relativistic energies, there is a basic interest in pion physics, particularly with respect to interactions in excited nuclear matter. In addition, it is believed that pion emission patterns can be used in the study of heavy ion reaction mechanisms. Whether viewed as proceeding by a series of independent nucleon-nucleon collisions, ${ }^{1,2}$ or at the other extreme, as a thermal process, ${ }^{3-5}$ pion production in heavy ion collisions occurs in the most violent stage of the reaction in a geometrical overlap or "participant" region. Because of the relatively large amount of energy needed to produce a pion, as compared to the energy necessary to liberate a nucleon from a nucleus, the pion source should be well localized at bombarding energies used in the present study.

Subsequent processes such as absorption and Coulomb deflection can modify the pion emission patterns, depending upon the impact parameter of the heavy ion collision and the time of pion emis- sion, along with other properties of the reaction. Only $\pi^{+}$were measured in the experiments reported here, but it is apparent that a much more definitive study of reaction mechanisms would be possible if measurements were made for all three charge states of the pion.

In the present experiments ${ }^{6,7}$ performed at the LBL Bevalac, a systematic study of $\pi^{+}$production was made with 14 combinations of targets, projectiles, and bombarding energies. The projectile systematics was studied principally with $p,{ }^{4} \mathrm{He},{ }^{20} \mathrm{Ne}$, and ${ }^{40} \mathrm{Ar}$ at $1.05 \mathrm{GeV} / \mathrm{u}$ on a ${ }^{238} \mathrm{U}$ target, and the target dependence was investigated mainly with 400 $\mathrm{MeV} / \mathrm{u}{ }^{20} \mathrm{Ne}$ on targets of ${ }^{27} \mathrm{Al}, \mathrm{Ag},{ }^{197} \mathrm{Au}$, and ${ }^{238} \mathrm{U}$. For the pion production as a function of bombarding energy, the ${ }^{20} \mathrm{Ne}+{ }^{238} \mathrm{U}$ reaction was used at $E / A$ of $0.25,0.4,1.05$, and $2.1 \mathrm{GeV}$. Results from the ${ }^{40} \mathrm{Ar}+{ }^{40} \mathrm{Ca}$ reaction were compared at $E / A=0.4$ and $1.05 \mathrm{GeV}$. A further comparison is made with proton-nucleus data from other studies. The use of thin targets allowed good angular resolution, an important consideration for pions in the energy range of $15-95 \mathrm{MeV}$ as measured here with a large $\mathrm{Si}-\mathrm{Ge}$ telescope. Data were taken from $30^{\circ}$ to $150^{\circ}$, usually in $20^{\circ}$ steps. Particular attention was given to the accuracy of the absolute cross sections. Adding to the extensive nature of the study 
reported here, associated charged-particle multiplicity determinations were made. Multiplicity selections were performed on some of the $\pi^{+}$data (when the statistical accuracy allowed) in an attempt to set impact parameter restrictions (e.g., grazing or central collisions). Energy spectra of the $\pi^{+}$are presented as laboratory double differential cross sections in $10 \mathrm{MeV}$ bins, and as contours of the Lorentz invariant cross section as a function of perpendicular momentum and rapidity. This type of contour map or "rapidity plot" is often useful in locating sources of emission. Results are compared to a thermal model and to cascade calculations. Finally, simple multicenter Coulomb trajectory calculations were performed in an attempt to isolate Coulomb effects and to extract information on the reaction mechanisms. Although not as extensive as one might prefer, the comparison of the calculations with the data suggests that Coulomb effects may have been overemphasized in several calculations performed previously, and many of the features seen in the $\pi^{+}$-emission patterns demand other explanations.

\section{EXPERIMENTAL TECHNIQUES}

Pion data were collected with the same apparatus used for detection of protons, deuterons, and tritons as described elsewhere in detail. Briefly, pion energies were measured and particle identification was made with a multielement telescope consisting of a $5 \mathrm{~mm} \mathrm{Si}(\mathrm{Li})$ detector, followed by two intrinsic germanium crystals with thicknesses of 27 and $43 \mathrm{~mm}$. All of these detectors were nominally fully depleted. A $1 \mathrm{~mm} \mathrm{Si}(\mathrm{Li})$ counter behind the detector stack served as a veto for punch-through particles, chiefly pions above $100 \mathrm{MeV}$ and protons above $200 \mathrm{MeV}$. The solid angle of the telescope used for all pion cross section calculations was $0.88 \mathrm{msr}$, in agreement with later determinations of the solid angle with $\alpha$ sources which yielded a value of 0.85 $\mathrm{msr} \pm 4 \%$. Pion energies were corrected for target thickness $\left(109-200 \mathrm{mg} / \mathrm{cm}^{2}\right)$, for the cryostat window, and for a $150 \mu \mathrm{m}$ Si detector which acted as a $\Delta E$ counter for the heavier particles, but only as an absorber for the pion data. A value of $4.2 \mathrm{MeV}$ was subtracted from apparent $\pi^{+}$energies to account for the muon energy from the $\pi^{+} \rightarrow \mu^{+}+v$ decay. A useful pion energy range of $15-95 \mathrm{MeV}$ was attained, with the lower limit set by the requirement of traversal of the $5 \mathrm{~mm} \mathrm{Si}(\mathrm{Li})$ detector into the intrinsic Ge, and deposition of several MeV. Approximately $3 \mathrm{MeV}$ on either end of the pion spectrum was not used to avoid efficiency losses due to positron escape, as discussed later. Pion energy bins were chosen such that small discontinuities in spectra that occur due to dead layers of the germanium detectors would average out, which allowed energy bins of $10 \mathrm{MeV}$ width centered from 20 to $90 \mathrm{MeV}$.

The telescope was operated in a cryostat situated in an evacuated spherical scattering chamber of $1 \mathrm{~m}$ diameter. A charged-particle multiplicity array consisting of 80 plastic scintillators (of $6.4 \mathrm{~mm}$ thickness) coupled to photomultiplier tubes was situated just outside of the scattering chamber. This array, which was triggered by the telescope, registered charged particles which penetrated the 3 $\mathrm{mm}$ aluminum chamber wall, corresponding to energies greater than $10 \mathrm{MeV}$ for $\pi^{+}$and $25 \mathrm{MeV}$ for protons. The array was inefficient for neutron detection because of the use of thin plastic scintillators. Only 76 of the paddles were used in the pionassociated multiplicity analysis, with four paddles situated at backward angles being omitted. Approximately $66 \%$ of the hemisphere forward of the target was covered by the array situated at polar angles of $9^{\circ}$ to $80^{\circ}$.

The 14 target-projectile combinations studied here are listed in Table I, along with the nominal and actual beam energies entering the targets, corrected for material in the beam line.

\section{A. Pion identification in the telescope}

Charged pions in the telescope were distinguished by $d E / d X$ from other charged particles, $e^{ \pm}, K^{+}, p$, $d, t$, etc. present in the reaction products, but no attempt was made to separate $\pi^{+}$from $\mu^{+}$. In the analysis of the event-by-event data, each successive pair of detectors in the stack was used in the appropriate energy range as a $\Delta E-E$ telescope, with the next detector in the stack serving as a reject counter. With this procedure, charged pions were adequately resolved from other products, except at the most forward angles $\left(20^{\circ}, 30^{\circ}\right)$ or at low bombarding energies where the proton to pion ratio is large, $\geq 10^{3}$. Pileup effects and nuclear reactions occurring in the detectors caused this limitation through a continuous background of events in the $\Delta E-E$ space. With $d E / d X$ techniques alone, both $\pi^{+}$and $\pi^{-}$are included, even though the stopped pions of the two charge states interact very differently with the surrounding matter. Negative pions capture in $\mathrm{Ge}$ nuclei, forming stars, and sometimes the kinetic energy information of the $\pi^{-}$ 
TABLE I. Projectiles, nominal and corrected bombarding energies per nucleon, projectile rapidities $(y)$, and targets for the systems studied. Target thicknesses in $\mathrm{mg} / \mathrm{cm}^{2}$ are given in parentheses.

\begin{tabular}{|c|c|c|c|c|c|c|c|c|}
\hline Projectile & $\begin{array}{c}\text { Nominal energy } \\
(\mathrm{MeV})\end{array}$ & $\begin{array}{c}\text { Corrected energy } \\
(\mathrm{MeV})\end{array}$ & $y$ & $\begin{array}{c}\mathrm{Al} \\
(109)\end{array}$ & $\begin{array}{c}\mathrm{Ca} \\
(200)\end{array}$ & $\begin{array}{c}\mathrm{Ag} \\
(185)\end{array}$ & $\begin{array}{c}\mathrm{Au} \\
(197)\end{array}$ & $\underset{(200)}{\mathbf{U}}$ \\
\hline$P$ & 1050 & 1041 & 1.37 & & & & & $X$ \\
\hline \multirow[t]{2}{*}{${ }^{4} \mathrm{He}$} & 400 & 399 & 0.89 & $X$ & & & & $X$ \\
\hline & 1050 & 1049 & 1.38 & & & & & $X$ \\
\hline \multirow[t]{4}{*}{${ }^{20} \mathrm{Ne}$} & 250 & 241 & 0.70 & & & & & $X$ \\
\hline & 400 & 393 & 0.89 & $X$ & & $X$ & $X$ & $X$ \\
\hline & 1050 & 1045 & 1.38 & & & & & $X$ \\
\hline & 2100 & 2095 & 1.84 & & & & & $X$ \\
\hline \multirow[t]{2}{*}{${ }^{40} \mathrm{Ar}$} & 400 & 388 & 0.88 & & $X$ & & & \\
\hline & 1050 & 1042 & 1.37 & & $X$ & & & $X$ \\
\hline
\end{tabular}

is destroyed. From the study of $\pi^{ \pm}$spectra in this work and from direct measurements of the interaction of $\pi^{+}$and $\pi^{-}$beams in plastic scintillators, ${ }^{8}$ it was estimated that the products of $\pi^{-}$capture are extremely disruptive only about $15 \%$ of the time in the $\mathrm{Ge}$ crystals here. This is probably due to the mechanism of pion absorption, principally on two or a few nucleons ${ }^{9}$ which are then ejected from the nucleus and escape from the detector with high energies. These secondary nucleons deposit only a small amount of energy in the detector, still within the identification windows. Of course this feature is dependent upon the detector geometry.

Unambiguous $\pi^{+}$identification was made by following the subsequent muon decay in the Ge crystals through the decay sequence

$$
\begin{aligned}
& \pi^{+} \stackrel{26 \mathrm{~ns}}{\rightarrow} \mu^{+}+v, \\
& \mu^{+} \stackrel{2.2 \mu \mathrm{s}}{\rightarrow} e^{+}+v+\bar{v} .
\end{aligned}
$$

The $e^{+}$was detected in delayed coincidence with the $\pi^{+}$signal, resulting in a decay curve with a characteristic $2.2 \mu$ s mean lifetime. An example of a time spectrum from a time-to-amplitude converter is shown in Fig. 1. The number of chance events became significant at forward angles and at a relatively high beam intensity. The magnitude was determined by fitting the decay curve with a twocomponent function, corresponding to $\tau=2.2 \mu \mathrm{s}$ and a random background $(\tau=\infty)$. This procedure was found to have a very small uncertainty and proved to be more reliable than the use of the more common method which employs the time region 1 $\mu \mathrm{s}$ ahead of the position of the prompt peak. The latter time region was subject to contributions from overshoot of very large prompt pulses (from $p, d, t$, etc.), which contaminated the time spectra $\pm 1 \mu \mathrm{s}$ from $t=0$ and which therefore was not random.

For the same reason, $\pi^{+}$data were used only for times greater than $1 \mu \mathrm{s}$. It was confirmed that chance-subtracted $\pi^{+}$energy spectra proved to be independent of time in the region used, corresponding to $t>1 \mu \mathrm{s}$. The loss of the time interval $0-1$ $\mu$ s caused a large $\pi^{+}$efficiency loss and precluded any hope of obtaining accurate $\pi^{-}$information by subtracting the $\pi^{+}$data. Figure 1(b) shows an energy spectrum for delayed $e^{+}$in the Ge crystals. The spectrum is degraded because of the relatively small volume of the detector, compared to the amount of material that would be necessary to stop the $e^{+}$, which are emitted with energies up to $E_{\max }=53 \mathrm{MeV}$ and with a spectral shape

$$
P(W)=2 W^{2}(3-2 W), W=E_{e} / E_{\max } .
$$

The above spectral shape indicates that the probability of emission of $e^{+}$with an energy lower than the $1 \mathrm{MeV}$ level of the constant fraction discriminator used here is extremely low.

\section{B. Pion efficiency function}

The shape and magnitude of the $\pi^{+}$spectra must be corrected for efficiency losses as follows:

(1) For $\pi^{+}$stopped in the Ge crystals, the loss of $e^{+}$tag signals may occur because of insufficient energy deposition when muon decay occurs in the surface region of the Ge crystals. A loss of $3 \%$ was calculated from the $d E / d X$ of $e^{+}$in Ge, the known 

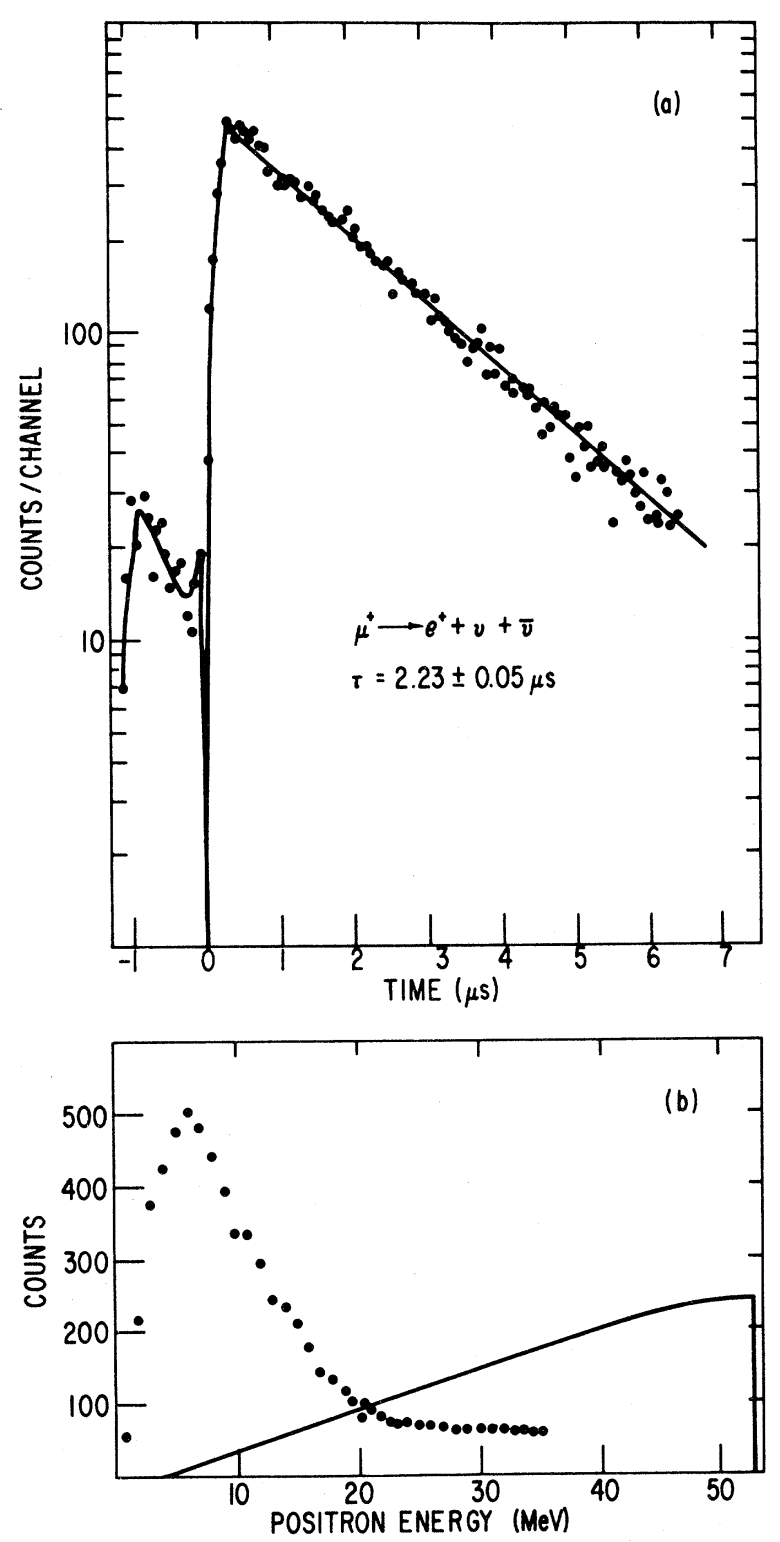

FIG. 1. (a) Time spectrum taken from the 1050 $\mathrm{MeV} / \mathrm{u}{ }^{40} \mathrm{Ar}+{ }^{40} \mathrm{Ca}$ reaction showing the characteristic $2.2 \mu \mathrm{s}$ mean lifetime of muon decay. The location of the prompt peak, suppressed with a 100 ns anticoincidence pulse, is at $t=0$. (b) Data points represent the energy spectrum of delayed coincidence pulses attributed to $e^{+}$from muon decay, and the curve is the theoretical shape $P(W)$.

spectral shape of $e^{+}$, and assuming a uniform distribution of $\pi^{+}$in the Ge crystals.

(2) The decay in flight of $\pi^{+}$before reaching the telescope is a negligibly small correction because of the short flight path $(25 \mathrm{~cm})$ from the target. Also since $\mu^{+}$are included with $\pi^{+}$in the spectra, ap- proximately as many $\mu^{+}$are deflected into the telescope as are deflected out, with negligible smoothing of the angular distribution. The unimportance of this correction is an advantage of the rangeenergy method of pion detection over magnetic analysis.

(3) The $\pi^{+}$cross section was corrected for a $45 \%$ efficiency loss incurred by using only $1-6 \mu \mathrm{s}$ of the decay curve. The uncertainty in this correction is negligible because time spectra were accurately calibrated. From these three corrections, the $\pi^{+}$efficiency is $53 \%$ and is nearly independent of pion energy.

(4) The correction that introduced the largest uncertainty in the data, especially at the highest pion energies, was for scattering-out of pions due to collision with atomic electrons during the stopping process. That is, $\pi^{+}$were deflected out of the edges of the telescope, depositing only part of their energy, and losing the delayed $e^{+}$tag event. The efficiency, defined as the fraction of surviving $\pi^{+}$, shown in Fig. 2 as curve A, was calculated with the Monte Carlo code ANGLE (Ref. 10) as described in Ref. 7. An experimental check of the validity of this calculation, together with the reaction loss correction, is described later.

(5) The loss of $\pi^{+}$through nuclear reactions in the detector was calculated by dividing the Ge crystals into ten slices, with $N_{i}$ atoms $/ \mathrm{cm}^{2}$ each, and by using range energy tables to calculate the $\pi^{+}$energy in each slice so that the detection efficiency $\epsilon$ is

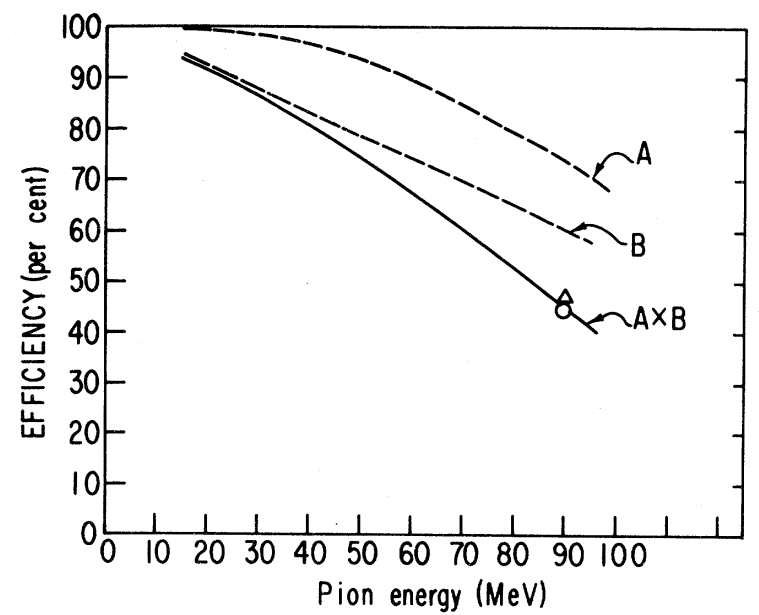

FIG. 2. Calculated telescope efficiency for $\pi^{+}$detection as a function of pion energy for scattering-out (A) and reaction loss $(\mathrm{B})$. The product of $\mathrm{A} \times \mathrm{B}$ was checked with $90 \mathrm{MeV}$ pions produced in the $1.05 \mathrm{GeV} / \mathrm{u}$ ${ }^{40} \mathrm{Ar}+{ }^{40} \mathrm{Ca}$ reaction (O) and the $1.05 \mathrm{GeV} / \mathrm{u} p+{ }^{238} \mathrm{U}$ reaction $(\Delta)$. 
given by

$$
\epsilon=\exp \left[-\sum_{i} N_{i} \sigma_{R}\left(E_{i}\right)\right] .
$$

Figure 2 shows the result of this calculation as curve B which gives a loss of efficiency of up to $27 \%$ at $95 \mathrm{MeV}$, the highest $\pi^{+}$energy used. Values of the $\pi^{+}$reaction cross sections as a function of energy $\sigma_{R}\left(E_{i}\right)$ were taken from a number of sources, including optical model calculations ${ }^{11}$ and measured absorption and inelastic scattering cross sections. ${ }^{12}$ The best estimate for $\sigma_{R}$ is believed to have been made by using experimental absorption cross sections $\sigma_{A}$ (which for Ge range from $350 \mathrm{mb}$ at $20 \mathrm{MeV}$ to $640 \mathrm{mb}$ at $100 \mathrm{MeV}$ ), and adding the large-angle part of the quasielastic and the charge exchange cross sections (which ranged from $15 \%$ of $\sigma_{A}$ at $20 \mathrm{MeV}$ to $60 \%$ of the $\sigma_{A}$ at $100 \mathrm{MeV}$ ), as calculated with the VEGAS code ${ }^{13}$ or from optical model codes. ${ }^{11}$

The uncertainties in the calibrated correction factors for scattering-out and for reaction loss are difficult to estimate. Since these factors affect the spectral shape, it was considered essential to make an experimental check on the efficiency curve. Fortunately, this was possible for pion energies of 70-100 MeV by performing a punch-through analysis of the spectra using data at backward angles, where pion spectra are free of background. For the most energetic pions that stop in the last thick element of the telescope ( $43 \mathrm{~mm} \mathrm{Ge})$, identification was made and the total energy was determined by using the first two detectors only [5 $\mathrm{mm}$ $\mathrm{Si}(\mathrm{Li})$ and $27 \mathrm{~mm} \mathrm{Ge]}$. The loss in the number of pions in the identification windows using the stopping counter, compared to the number from using only the first two counters, represents the effect of scattering-out and reaction loss in the $43 \mathrm{~mm} \mathrm{Ge}$ detector. For $90 \mathrm{MeV}$ pions, $\approx 90 \%$ of these losses of the whole telescope occur in this last element, thus making this method a sensitive test for pion energies with the largest corrections. This efficiency measurement is for a mixture of $\pi^{+}$and $\pi^{-}$as produced in the reaction with the $\pi^{-}$having a lower efficiency than the $\pi^{+}$. Approximately $15 \%$ of the $\pi^{-}$are lost in the capture process with the secondary particles depositing enough energy to remove the event from the particle identification windows. Also the absorption cross section is larger for $\pi^{-}$compared to $\pi^{+}$, e.g., by $25 \%$ at 83 and 100 MeV. ${ }^{12}$ Small corrections to the measured efficiency were made for these $\pi^{+}, \pi^{-}$differences in two cases where the initial $\pi^{+} / \pi^{-}$ratio was known. In the first case, pion emission at laboratory angles of $130^{\circ}$ and $150^{\circ}$ was used for $1.05 \mathrm{GeV} /$ nucleon ${ }^{40} \mathrm{Ar}+{ }^{40} \mathrm{Ca}$ since the $\pi^{+} / \pi^{-}$ratio at $100 \mathrm{MeV}$ was known to be nearly unity for the $800 \mathrm{MeV} / \mathrm{u}$ ${ }^{40} \mathrm{Ar}+\mathrm{KCl}$ reaction as determined by Nagayima et al. ${ }^{14}$ For the second case, the $1.05 \mathrm{GeV} p+{ }^{238} \mathrm{U}$ reaction at $90^{\circ}-150^{\circ}$ provided a pion source with a dominant contribution of $\pi^{+}$. A value for the $\pi^{+} / \pi^{-}$ratio of 3.5 was taken from the $p+{ }^{208} \mathrm{~Pb}$ reaction in the measurements of Cochran et al. ${ }^{15}$ These detector efficiency determinations are in good agreement with the calculated efficiencies as shown by the data points in Fig. 2. The estimated uncertainty of the cross section at $90 \mathrm{MeV}$, compared to $50 \mathrm{MeV}$ and below, is believed to be no more than $10 \%$. That is, there may be a systematic error in the shape of the $\pi^{+}$spectra such that in the data, features smaller than the $10 \%$ level probably are not significant. There is also a $20 \%$ uncertainty in the overall normalization of the data. ${ }^{7}$

\section{EXPERIMENTAL RESULTS}

\section{A. Single-particle inclusive data}

For pion production with medium-energy protons on heavy target nuclides, it is generally concluded that the $\pi^{+}$originate predominantly from protonproton scattering in the nuclear surface. ${ }^{16-18} \mathrm{~A}$ dependence on the target atomic number of $\approx Z^{1 / 3}$ (for $Z \gtrsim 15$ ) for the $\pi^{+}$production cross sections provides empirical evidence for surface production in data for 585,660 , and $730 \mathrm{MeV}$ protons. ${ }^{15,19,20}$ The calculations of Sternheim and Silbar ${ }^{18}$ show this to be only approximately true. For $730 \mathrm{MeV}$ protons on a heavy target such as ${ }^{208} \mathrm{~Pb}, \approx 75 \%$ of the emitted $\pi^{+}$originate from the diffuse density region $\left(\rho<0.9 \rho_{0}\right)$. One may expect a similar situation with heavy ions on a ${ }^{238} \mathrm{U}$ target as studied here. But in reactions involving relatively light target-projectile combinations such as ${ }^{4} \mathrm{He}+{ }^{27} \mathrm{Al}$, ${ }^{20} \mathrm{Ne}+{ }^{27} \mathrm{Al}$, and ${ }^{40} \mathrm{Ar}+{ }^{40} \mathrm{Ca}$, the pion spectra should be more representative of the whole production volume, and should not be dominated by pion absorption and scattering. Single pion inclusive data for these three light nuclear systems at 400 $\mathrm{MeV} / \mathrm{u}$ are shown in Fig. 3. The double differential cross sections are plotted on semilogarithmic scales as a function of pion kinetic energy. All of the spectra are continuous, gently peaked distributions, and the laboratory angular distributions are nearly isotropic. This may be contrasted to free $p+p$ pion production which shows prominent structure in the energy spectra and a forward-backward directed 

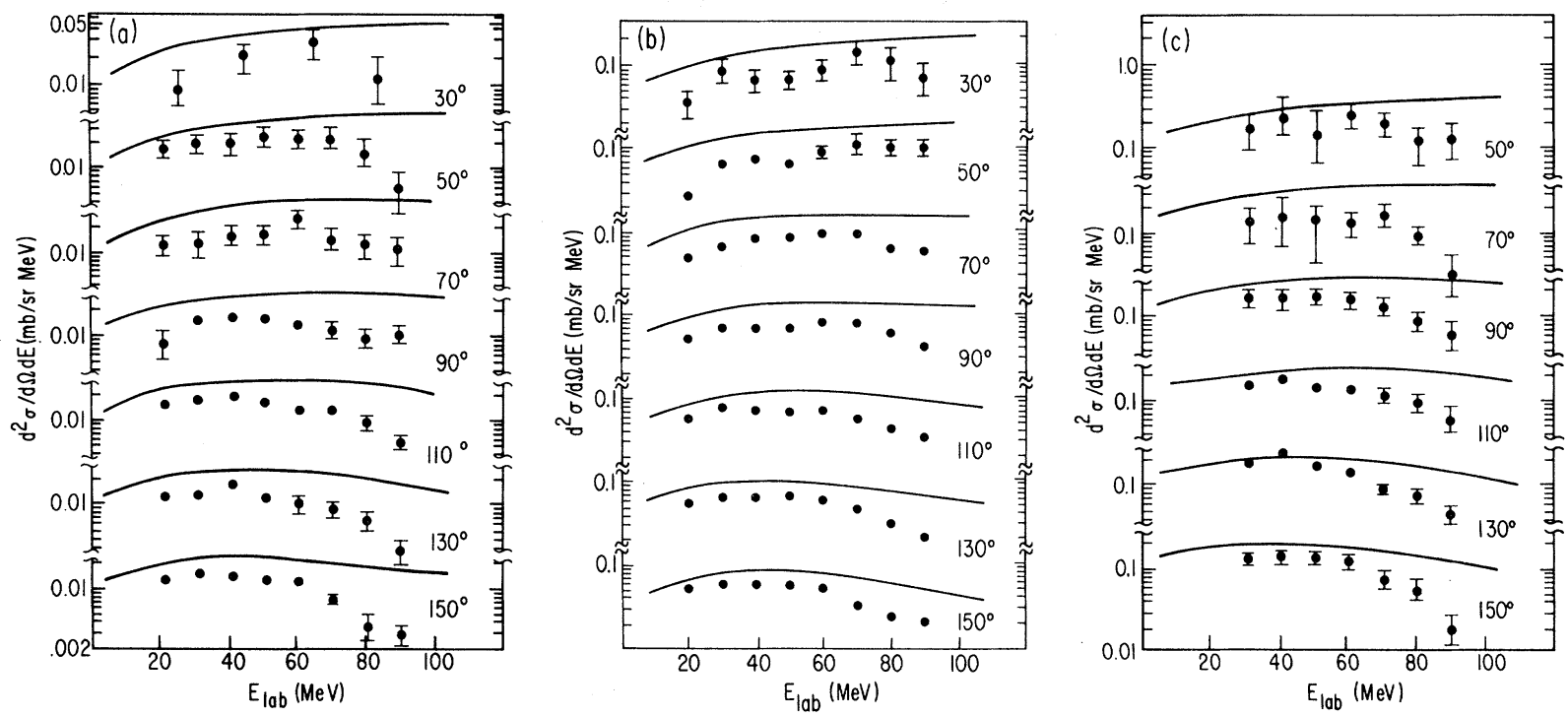

FIG. 3. Laboratory $\pi^{+}$inclusive cross section data for $400 \mathrm{MeV} / \mathrm{u}$ for the projectile-target combinations (a) ${ }^{4} \mathrm{He}+{ }^{27} \mathrm{Al}$, (b) ${ }^{20} \mathrm{Ne}+{ }^{27} \mathrm{Al}$, and (c) ${ }^{40} \mathrm{Ar}+{ }^{40} \mathrm{Ca}$. The solid curves show the results of the nuclear firestreak models.

c.m. angular distribution, chiefly due to the $p+p \rightarrow \pi^{+}+d$ reaction at low bombarding energies. ${ }^{21}$ The smoothness of the spectra and the isotropy of the angular distributions for heavy ion reactions may be attributed to the intrinsic nucleon motion and, to a lesser extent, pion scattering and Coulomb effects. The Fermi motion is quite important for pion production close to threshold. In a free nucleon-nucleon collision at $400 \mathrm{MeV}$ there is only $\approx 50 \mathrm{MeV}$ of extra energy above pion threshold in the center of mass. Thus in heavy ion reactions, a Fermi energy of $\approx 30-40 \mathrm{MeV}$ and a high momentum tail in both the target and the projectile cause the pion production cross section to be greatly enhanced near the free nucleon-nucleon threshold. Moreover, the energy spectra and angular distributions are smeared through interactions, analogous to the $p+p \rightarrow \pi^{+}+d$ reaction, but with coupling to the Fermi motion instead of the deuteron final state. By the same arguments, one expects harder, flatter pion spectra in heavy ion reactions than in proton plus nucleus reactions, which seems to be the case in comparison of Fig. 3 to $\pi^{+}$data taken for 380 and $450 \mathrm{MeV}$ protons on targets of ${ }^{12} \mathrm{C}$ and $\mathrm{Cu}^{22-25}$

Well above pion threshold, in the $1 \mathrm{GeV} / \mathrm{u}$ range, Fermi motion considerations should be less important and are expected to even decrease pion production cross sections. ${ }^{26}$ One expects forwardbackward peaked angular distributions in the nucleon-nucleon center of mass, and broad peaks in the pion energy spectra, corresponding to
$E_{\text {c.m. }}^{*} \approx 100-200 \mathrm{MeV}$ caused by isobar decay as observed in proton-induced ${ }^{27,28}$ and neutroninduced $^{29}$ reactions on light nuclides. This characteristic signature of isobar decay seems to occur in heavy ion reactions, but with additional features, especially at low pion energies. The previously reported results for the $1.05 \mathrm{GeV} / \mathrm{u}{ }^{40} \mathrm{Ar}+{ }^{40} \mathrm{Ca}$ reaction, ${ }^{6}$ from the present study as well as data for the $800 \mathrm{MeV} / \mathrm{u}{ }^{20} \mathrm{Ne}+\mathrm{NaF}$ reaction, ${ }^{30,31}$ are representative examples. The pion spectra for the 1.05 $\mathrm{GeV} / \mathrm{u}{ }^{40} \mathrm{Ar}+{ }^{40} \mathrm{Ca}$ reaction are shown in Fig. 4 in linear plots along with results from three calculations. The thin-lined histogram shows results of an intranuclear cascade code of Yariv and Fraenkel ${ }^{1}$ (called cascade I here), which tests some of the qualitative arguments made regarding pion production from a superposition of nucleon-nucleon collisions. The Fermi motion of the target and the projectile is included and the isobar model is used for production and absorption as treated by Harp. ${ }^{32}$ It can be seen in Fig. 4 that this calculation differs from the data in a more fundamental way than by a mere absolute normalization factor. While the calculation falls below the data at $\theta_{\text {lab }}=30^{\circ}$ corresponding to approximately $80^{\circ}-90^{\circ}$ in the center of mass, too much yield is predicted at backward angles. This is apparently caused by the forwardbackward directed c.m. angular distribution of the isobar decay which has not been fully damped out in cascade I. The difference is further magnified by the fact that the data show an actual sidewise ejection of pions of low $E_{\text {c.m. }}$. A more isotropic angu- 


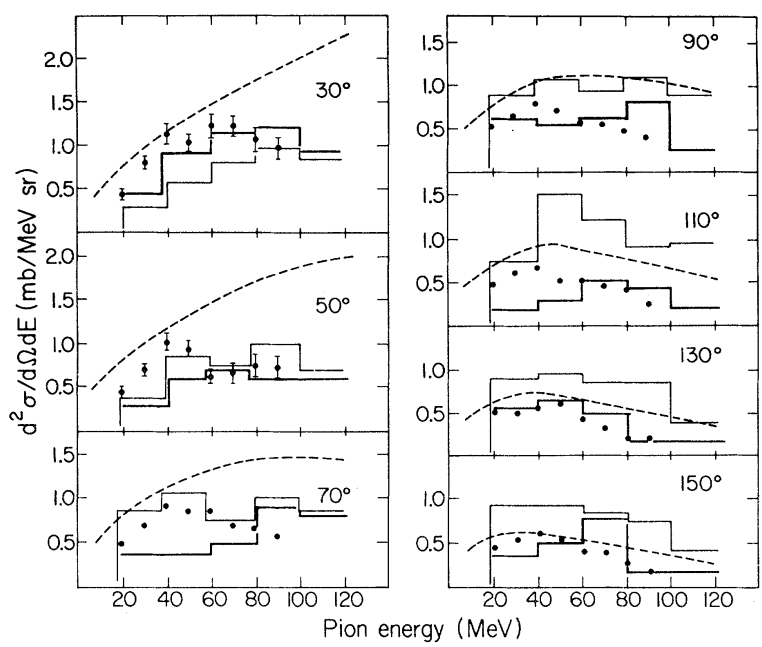

FIG. 4. Pion-inclusive double differential cross sections in the laboratory system for the reaction ${ }^{40} \mathrm{Ar}+{ }^{40} \mathrm{Ca} \rightarrow \pi^{+}+X$ at $1.05 \mathrm{GeV} / \mathrm{u}$. The histograms (solid lines) show the results of two intranuclear cascade calculations (Refs. 1 and 2) and the dashed lines represent a nuclear firestreak calculation (Ref. 5).

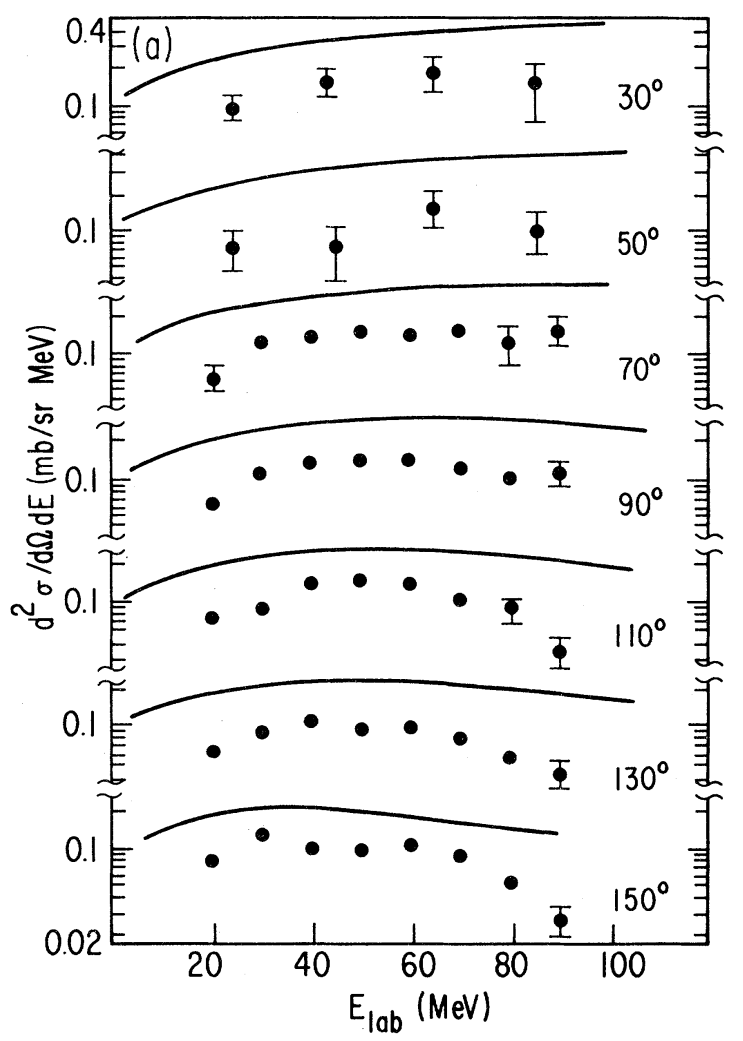

lar distribution, and a better fit to the data, is given by the bolder histogram, which represents an improved cascade calculation performed recently by Yariv and Fraenkel ${ }^{2,33}$ (cascade II). The most important addition to cascade $I$ is the inclusion of cascade-cascade interactions, i.e., the scattering of particles which were promoted out of the Fermi sea in previous interactions. Effectively, increased thermalization is allowed with a more complete loss of the directional memory of the entrance channel in the isobar decay, as well as increased scattering and annihilation of isobars and pions. Such effects are expected to be important in heavy ion reactions, because of the high density of interactions. The agreement of cascade II with experiment is good in this instance, considering that the Coulomb effects on the pion emission probabilities and trajectories have not been included.

Calculated results from a thermal model, the firestreak model, ${ }^{5}$ are shown by the dashed line in Fig. 4 and by the solid lines in Fig. 3 and Figs. 5-7. As a general observation, the cross sections predicted

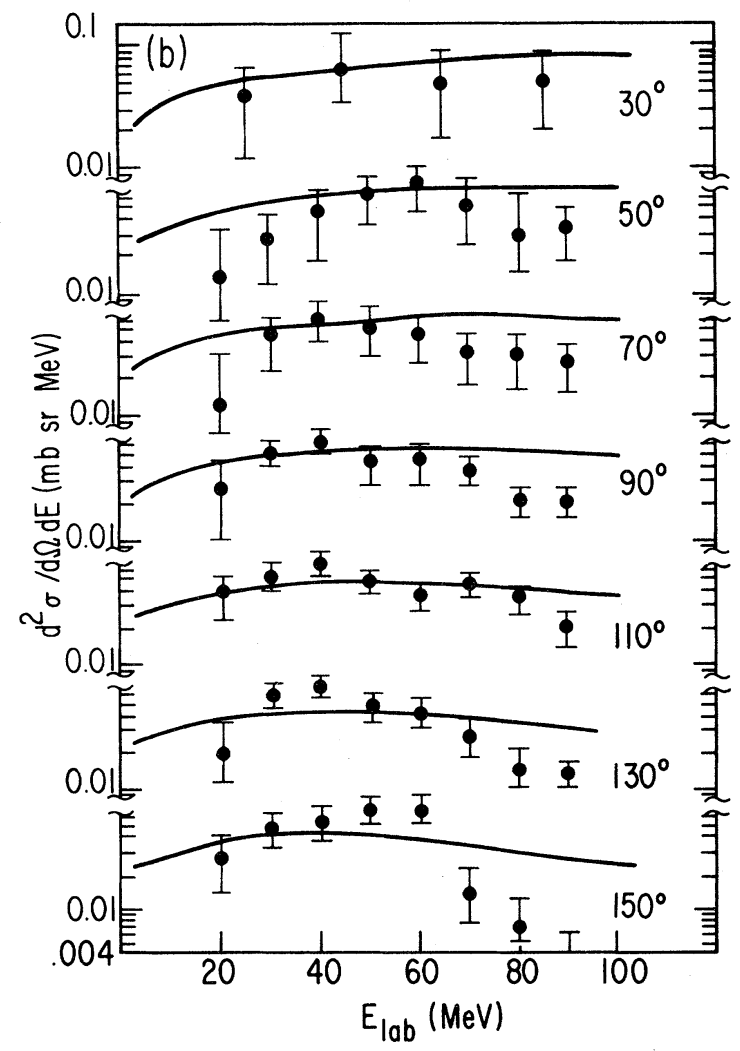

FIG. 5. Same as Fig. 3 except the target-projectile combinations are $400 \mathrm{MeV} / \mathrm{u}$ (a) ${ }^{20} \mathrm{Ne}+{ }^{107,109} \mathrm{Ag}$ and (b) ${ }^{4} \mathrm{He}+{ }^{238} \mathrm{U}$. 

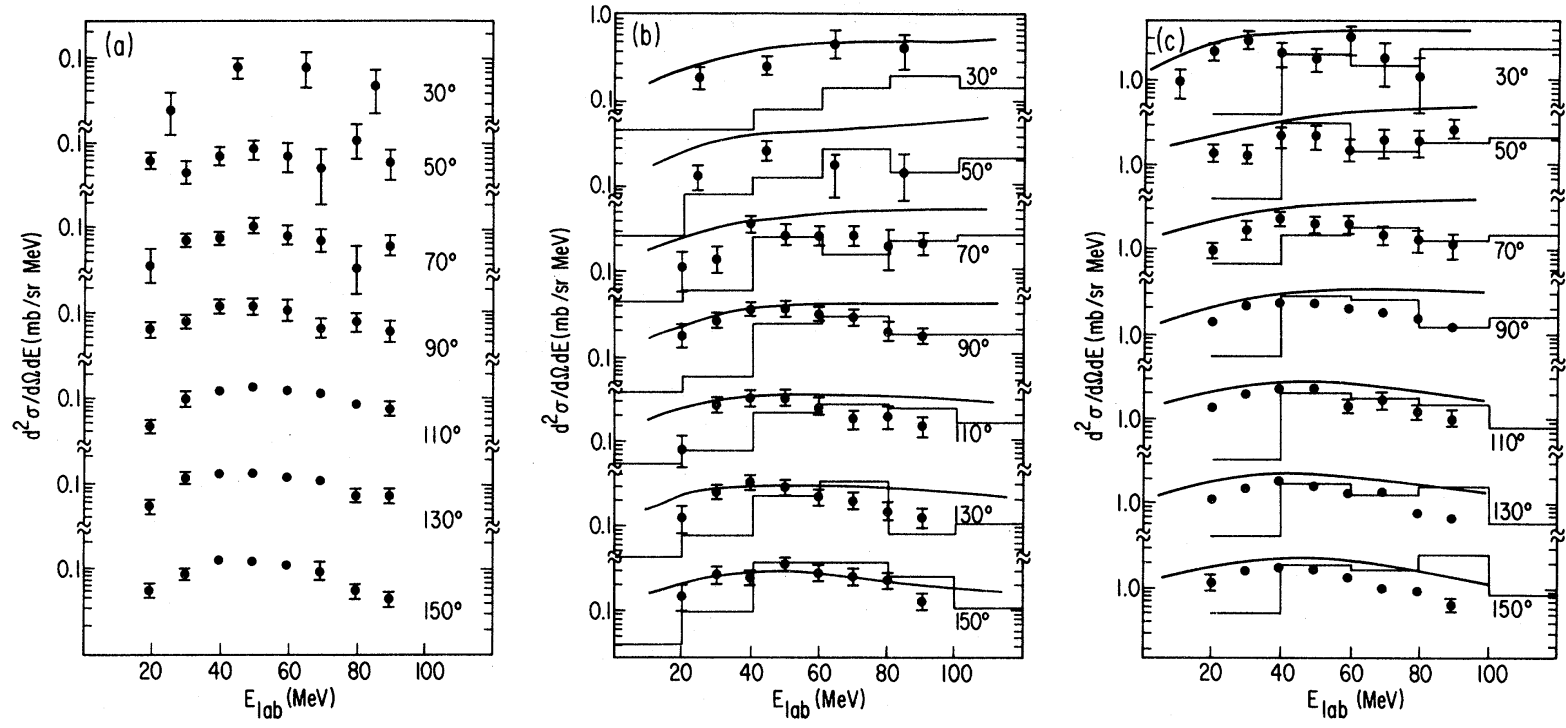

FIG. 6. Same as Fig. 3 except at $1.05 \mathrm{GeV} / \mathrm{u}$ (a) $p+{ }^{238} \mathrm{U}$, (b) ${ }^{4} \mathrm{He}+{ }^{238} \mathrm{U}$, and (c) ${ }^{40} \mathrm{Ar}+{ }^{238} \mathrm{U}$.

by the firestreak calculations are $\approx 50 \%$ too high, and the spectra are too hard. That is, the high energy tails of the data fall off much faster than the calculation. There are several shortcomings in this version of the firestreak calculation, such as the lack of Coulomb effects, and the neglect of pion scattering and absorption in the spectator matter. A more fundamental restriction in the "fire" models is imposed by the assumption of complete thermalization, which is not supported by intranuclear cascade calculations. ${ }^{1}$ For light systems such as ${ }^{12} \mathrm{C}+{ }^{12} \mathrm{C}$ or ${ }^{20} \mathrm{Ne}+{ }^{20} \mathrm{Ne}$, even for central impact parameters, there are less than two collisions per participant nucleon on the average. For heavy systems, nucleon-nucleon collisions in the nuclear surface should provide a component of promptly emitted pions from isobar decay $\left(\tau \approx 0.7 \times 10^{-23} \mathrm{~s}\right)$ which will not obey the predictions of the thermal models. Also this pion emission will have a cooling effect which causes subsequent processes to have an effectively lower temperature. With these considerations, at best only approximate agreement with the inclusive data is expected in light or heavy systems, which seems to be the case. The bombarding energy dependences of the cross section magnitudes predicted by the firestreak calculations are reasonably well reproduced, e.g., for four energies in the ${ }^{20} \mathrm{Ne}+{ }^{238} \mathrm{U}$ reaction shown in Fig. 7, from $E / A$ of $250 \mathrm{MeV}$ (below the free $p+p$ threshold) to 2.1 $\mathrm{GeV}$, with an increase in the pion production cross section of two orders of magnitude.

\section{B. Associated charged-particle multiplicities}

A more complete presentation of the multiplicity measurements made in this study is given in Ref. 7 . Pion-associated charged-particle multiplicities are compared briefly here with the data of Ref. 7 which dealt with $p, d, t$ triggering of the same 80-paddle array. The major emphasis of the present work is in the use of the multiplicity data to set impact parameter restrictions on the pion spectra presented in the preceding section. Two examples of charged-particle multiplicity distributions are shown in Fig. 8 for the reactions ${ }^{20} \mathrm{Ne}+{ }^{238} \mathrm{U}$ and ${ }^{40} \mathrm{Ar}+{ }^{238} \mathrm{U}$, each at a total kinetic energy of 42 $\mathrm{GeV}$. These are raw data, uncorrected for incomplete solid angle coverage and coincidence summing. Thus an observed average multiplicity $\langle m\rangle$ of 25 for each of these two cases attains a value of 52 for the average multiplicity $\langle M\rangle$ with the corrections. For reasons discussed in Ref. 7, due to possible correlations of charged particles, only the corrected average values are extracted and higher moments are not obtained. It should be emphasized that these are associated multiplicity distributions, triggered by a pion detected in the $\mathrm{Si}-\mathrm{Ge}$ telescope at $90^{\circ}$ in the laboratory, and not unbiased multiplicities. In most instances, the average associated multiplicities $\langle\boldsymbol{M}\rangle$ are approximately a factor of 2 higher than the unbiased charged particle multiplicities as determined from the inclusive data and the 

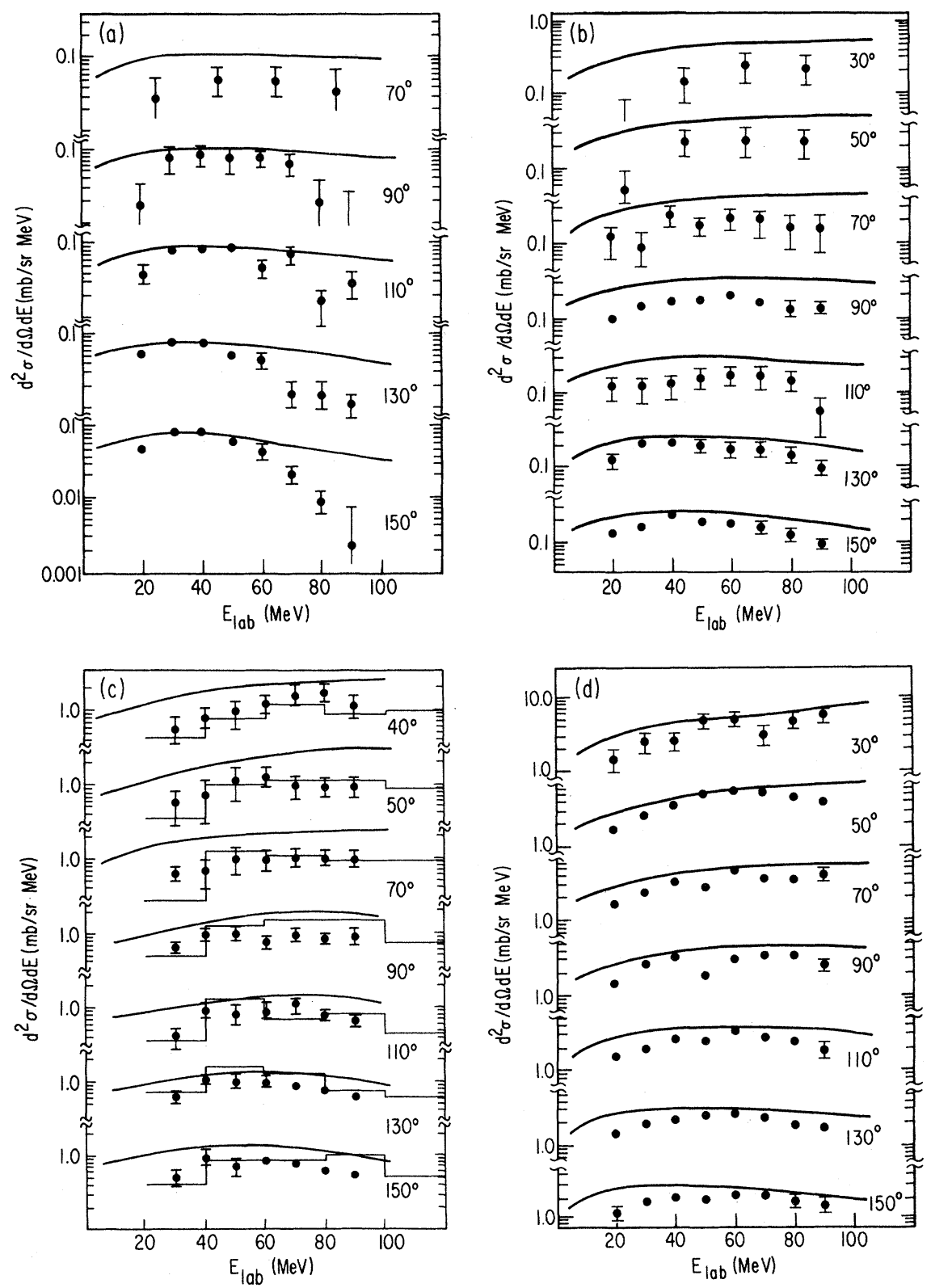

FIG. 7. Same as Fig. 3 except the target-projectile combination is ${ }^{20} \mathrm{Ne}+{ }^{238} \mathrm{U}$ at (a) $250 \mathrm{MeV} / \mathrm{u}$, (b) $400 \mathrm{MeV} / \mathrm{u}$, (c) $1.05 \mathrm{GeV} / \mathrm{u}$, and (d) $2.1 \mathrm{GeV} / \mathrm{u}$.

geometric reaction cross section. Apparently the detection of a $\pi^{+}$(or $\left.p, d, t\right)$ at large angles discriminates against peripheral collisions. Another feature demonstrated by the two examples in Fig. 8 is the apparent scaling of the average associated multiplicity with the total kinetic energy of the projectile, as shown previously for proton-associated multiplicities. $^{7}$ Higher moments of the distribution do differ here, unlike the proton associated distributions which were identical within the statistical uncer- tainties. The values of the average associated multiplicities $\langle M\rangle$ generally show only small variations with respect to detection angle and particle type $\left(\pi^{+}, p, d, t\right)$. Figure 9 compares proton- and piontriggered values of $\langle M\rangle$ for $1.05 \mathrm{GeV} / \mathrm{u}{ }^{40} \mathrm{Ar}$ ions on targets of ${ }^{40} \mathrm{Ca}$ and ${ }^{238} \mathrm{U}$. For lighter targetprojectile combinations such as ${ }^{20} \mathrm{Ne}+{ }^{27} \mathrm{Al}$ and ${ }^{40} \mathrm{Ar}+{ }^{40} \mathrm{Ca}$, the values of $\langle M\rangle$ are constant with respect to the $\pi^{+}$-triggering angle, which probably indicates that most pions originate from a single 


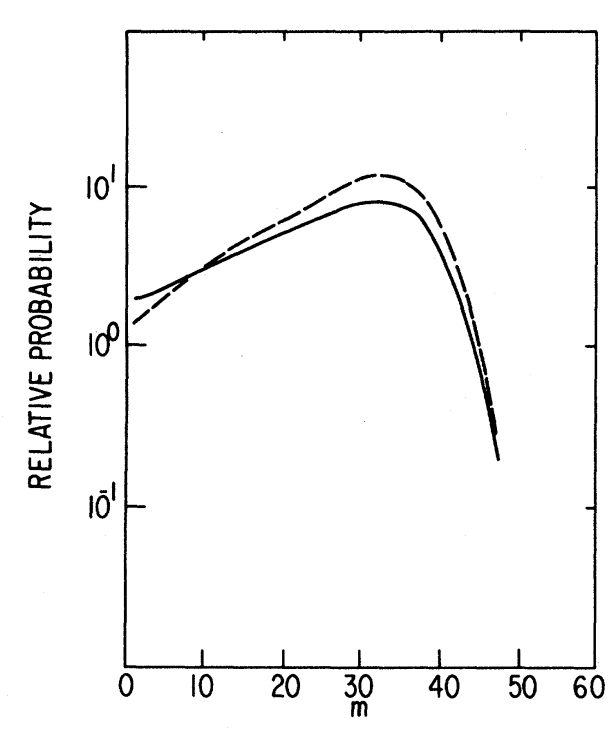

FIG. 8. Coincidence probability distributions for 1.05 $\mathrm{GeV} / \mathrm{u}{ }^{40} \mathrm{Ar}$ and $2.1 \mathrm{GeV} / \mathrm{u}{ }^{20} \mathrm{Ne}$ on a ${ }^{238} \mathrm{U}$ target. The abscissa is the number of scintillation paddles fired in the 80-paddle array, when triggered by a $15-95 \mathrm{MeV}$ $\pi^{+}$detected at $90^{\circ}$ in the laboratory.

type of reaction throughout the reaction volume. Note that this result disagrees with the predictions of Ref. 2, in which central collisions should be enhanced for a back-angle emitted $\pi^{+}$. The proton-associated multiplicities, on the other hand, are somewhat smaller and fall off significantly at backward angles, which may be interpreted in terms of contributions from spectator deexcitation, that is, nucleons ejected from less violent processes in the spectator matter. For the ${ }^{40} \mathrm{Ar}+{ }^{238} \mathrm{U}$ reaction, one can see in Fig. 9 that the $\pi^{+}$and proton-associated

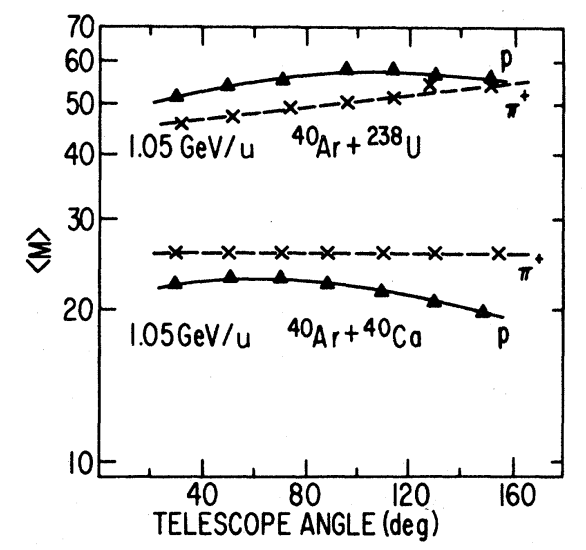

FIG. 9. Average associated multiplicities (corrected) as a function of laboratory angle for proton and $\pi^{+}$ triggers for $1.05 \mathrm{GeV} / \mathrm{u}{ }^{40} \mathrm{Ar}$ on targets of ${ }^{40} \mathrm{Ca}$ and ${ }^{238} \mathrm{U}$. multiplicities are nearly equal, and $\langle M\rangle$ continuously increases with increasing $\pi^{+}$detection angle. These rather different properties compared to light systems can be qualitatively understood with the arguments given in Sec. III A for nucleon-induced $\pi^{+}$ production, in terms of attenuation of incoming nucleons and outgoing pions. With a heavy target, calculations ${ }^{17}$ indicate $\approx 50 \%$ of the pion production originates on the nuclear surface $\left(\rho<0.9 \rho_{0}\right)$ from an annular region with the beam direction being the symmetry axis, i.e., moderately large impact parameters. Thus there is a bias toward lower charged-particle multiplicity events (as long as the $\pi^{ \pm}$emission is not a dominant contribution to $\langle M\rangle$ ). In this way the $\langle M\rangle$ are lowered for $\pi^{+}$to values which are comparable to proton-triggered events. The higher multiplicity at backward angles is caused again by surface production of the $\pi^{+}$, preferentially on the upstream side of the nucleus, due to degradation of incident nucleon energies below the pion threshold as the ${ }^{40} \mathrm{Ar}$ penetrates the ${ }^{238} \mathrm{U}$ nucleus in central or near central collisions.

It has been and will continue to be assumed that there is a close correspondence between the impact parameter of a collision and the charged-particle multiplicity as measured here. Although there is some experimental justification, as reviewed by Stock, ${ }^{35}$ for a monotonically increasing multiplicity with decreasing impact parameter, the actual basis for this relationship resides in using the number of participant nucleons in the fireball model, and in intranuclear cascade calculations. At this time, the assignment of the impact parameter range to an associated multiplicity can be made only loosely. Consequently the impact parameter restrictions made in the next section are of a qualitative nature. In the future it should be possible to simulate experimental conditions (energy cutoffs, efficiencies, and trigger bias) in an intranuclear cascade calculation. The cascade I calculation of Yariv and Fraenkel ${ }^{1}$ is useful for understanding some features of the correspondence between $\langle M\rangle$ and the impact parameter, although there are indications from the present work and from streamer chamber data ${ }^{36}$ that for reactions such as ${ }^{40} \mathrm{Ar}+{ }^{40} \mathrm{Ca}$, the predicted multiplicity is too low for small impact parameters. This calculation does demonstrate that the ideas of a participant volume are approximately correct, with the maximum sensitivity for impact parameter selection occurring for heavy systems with equal target and projectile masses as shown much earlier by the fireball model. An important point made in these calculations is the requirement of sufficient 
mass in the composite system to avoid fluctuations in the multiplicity for a given impact parameter, which are large enough to essentially invalidate the selection. Thus for ${ }^{4} \mathrm{He}+{ }^{27} \mathrm{Al}$ and ${ }^{20} \mathrm{Ne}+{ }^{27} \mathrm{Al}$ we expect only a very crude impact parameter bias with multiplicity gating, but for ${ }^{40} \mathrm{Ar}+{ }^{40} \mathrm{Ca}$ and heavier masses there should be sufficient sensitivity. Selection levels in Sec. III C are generally dictated by statistical considerations, and were usually made for $20 \%$ of the cross section with the lowest and highest multiplicities for the $90^{\circ}$ spectra.

\section{Rapidity plots and} multiplicity selected data

In subsection A of this section, single particle inclusive cross sections were compared to several calculations. Viewing the data in this manner, i.e., always with respect to a model, is somewhat limited, especially if none of the calculations contains the necessary physics to explain certain features. A more model-independent way of greater sensitivity for presenting the cross sections is in terms of socalled "rapidity plots" as described in more detail elsewhere. ${ }^{7}$ Contours of a constant (relativistically invariant) cross section, as a function of perpendicular momentum (divided by the mass of the particle) on the vertical axis and rapidity

$$
\left(y=0.5 \ln \left[\left(E_{T}-P_{\|}\right) /\left(E_{T}+P_{\|}\right)\right]\right)
$$

on the horizontal axis can be used to identify sources of emitted particles, such as target, projectile, nucleon-nucleon, or fireball reference frames. A simple translation along the rapidity axis in Galilean fashion allows one to shift between different coordinate systems. In the nonrelativistic limit the axes of these contour diagrams reduce to velocity components parallel and perpendicular to the beam axis. Figure 10(a) is an example of a rapidity plot using pion-inclusive cross sections for $\mathbf{4 0 0}$ $\mathrm{MeV} / \mathrm{u}{ }^{4} \mathrm{He}$ ions on a target of ${ }^{27} \mathrm{Al}$, the lightest system in this study. The small open circles mark the positions of the laboratory cross sections from Fig. 3(a), and show the angular intervals $\left(20^{\circ}\right)$ of data points used in construction of all of the contour diagrams to follow, except for the $1.05 \mathrm{GeV} / \mathrm{u}$ ${ }^{20} \mathrm{Ne}+{ }^{238} \mathrm{U}$ reaction, where data were taken every $10^{\circ}$. To minimize the influence of statistical fluctuations, the spacing of the contour lines was required to be greater than one standard deviation in the data. Since the topography of the pion emission is generally quite flat in the region investigated here, linear spacing of the contour lines was chosen.
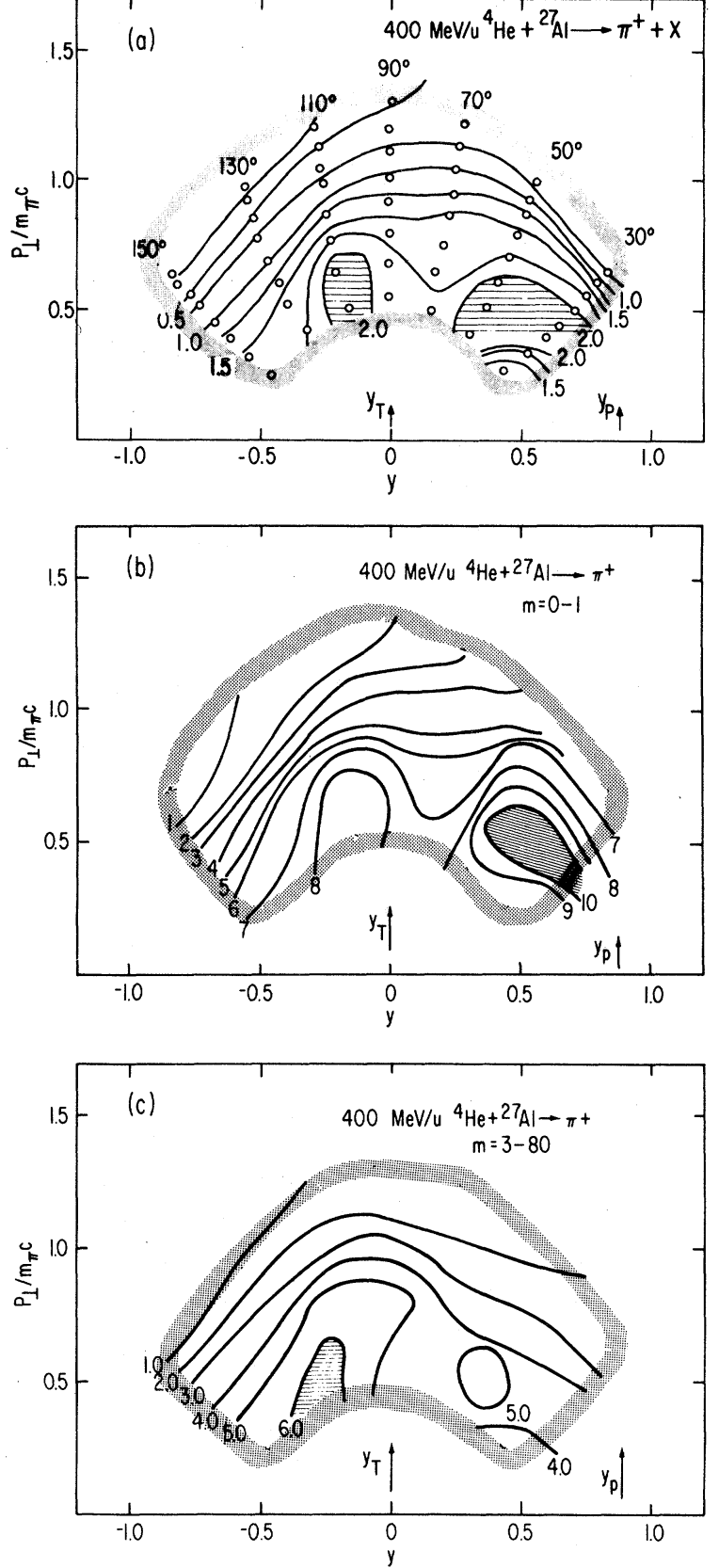

FIG. 10. Contours of the constant Lorentz-invariant pion cross section, $(1 / p) d^{2} \sigma / d \Omega d E$, in units of $\mathrm{b} /\left(\mathrm{sr} \mathrm{GeV}^{2} / c\right) \times f$ as a function of perpendicular pion momentum and rapidity $y$ for the $400 \mathrm{MeV} / \mathrm{u}$ ${ }^{4} \mathrm{He}+{ }^{27} \mathrm{Al}$ reaction. The areas within the maximum contours have horizontal shading. The shaded edges indicate the limits of the experimental measurements and $y_{P}$ and $y_{T}$ denote the projectile and target rapidities. (a) The pion inclusive cross sections. The laboratory data points are shown as open circles, with the corresponding laboratory angles noted, $f=10^{-4}$. (b) Same as (a) except gated on low charged-particle multiplicity, $f=10^{-5}$; (c) gated on high multiplicity, $f=10^{-5}$. 
A spline-interpolation method ${ }^{37}$ for smoothing the data was used, with contour lines drawn both by hand and with an ANL 370 computer package, with good agreement throughout.

No simple emission pattern emerges for the ${ }^{4} \mathrm{He}+{ }^{27} \mathrm{Al}$ reaction in Fig. 10(a). If $\pi^{+}$emission were predominantly from free $p+p$ collisions, then the cross section contours should tend to be centered around $p_{\perp}=0, y=\left(y_{p}+y_{T}\right) / 2$, or at least be symmetric about a vertical line at $\left(y_{p}+y_{T}\right) / 2$, which is $90^{\circ}$ in the nucleon-nucleon center of mass. Instead, the contours at the largest values of momentum are centered much closer to the target rapidity, as predicted by the fireball model. The cross section maxima of low $p_{1}$, denoted by horizontal shading in Fig. 10, are the most prominent features of the spectra. The ridge at back angles, $110^{\circ}$ in the laboratory or approximately $135^{\circ}$ in the $N-N$ center of mass in the present case, is a common feature of nearly all the pion spectra presented here and elsewhere for heavy ion ${ }^{31}$ and hadronnucleus reactions. ${ }^{15,19}$ The enhanced $\pi^{+}$emission at large angles is apparently caused by a combination of (a) the forward-backward emission from isobars at rest in the $N N$ center of mass and (b) the Coulomb repulsion of the $\pi^{+}$from the targetlike fragment. Thus this ridge of cross section moves to more negative rapidities, away from $y_{T}$ as the target is increased in $Z$, as shown in Fig. 11(a) for 400 $\mathrm{MeV} / \mathrm{u}{ }^{20} \mathrm{Ne}$ on a variety of targets ranging from ${ }^{12} \mathrm{C}$ (Refs. 30 and 31 ) to ${ }^{238} \mathrm{U}$. Also shown is the effect of multiplicity gating for $1.05 \mathrm{GeV} / \mathrm{u}$ ${ }^{20} \mathrm{Ne}+{ }^{238} \mathrm{U}$, corresponding to the amount of the target fragment remaining. Results for $585 \mathrm{MeV}$ proton plus nucleus $\pi^{+}$production ${ }^{19}$ are shown in Fig. 11(b) for comparison. For the ${ }^{4} \mathrm{He}+{ }^{27} \mathrm{Al}$ reaction the cross section maximum in Fig. 10(a) at laboratory angles of $30^{\circ}-50^{\circ}$ (angles smaller than $90^{\circ}$ in the $N-N$ center of mass) is not so easily explained and is not a common feature in other systems. More information is provided in Figs. 10(b) and (c) with the results of low and high multiplicity selection, respectively, for ${ }^{4} \mathrm{He}+{ }^{27} \mathrm{Al}$. It can be seen that the forward-angle maximum is enhanced for peripheral collisions (low $M$ ). A similar behavior can be seen in Fig. 12 for $400 \mathrm{MeV} / \mathrm{u}{ }^{20} \mathrm{Ne}+{ }^{27} \mathrm{Al}$, but it is apparent that the relatively small separation of the target and projectile rapidities leads to a considerable superposition of effects. Another feature of the multiplicity selection shown in parts (c) of Figs. 10 and 12 is the increased population of the midrapidity region, $y \approx 0.45$, for central collisions, as expected in a thermal model. The differences in the
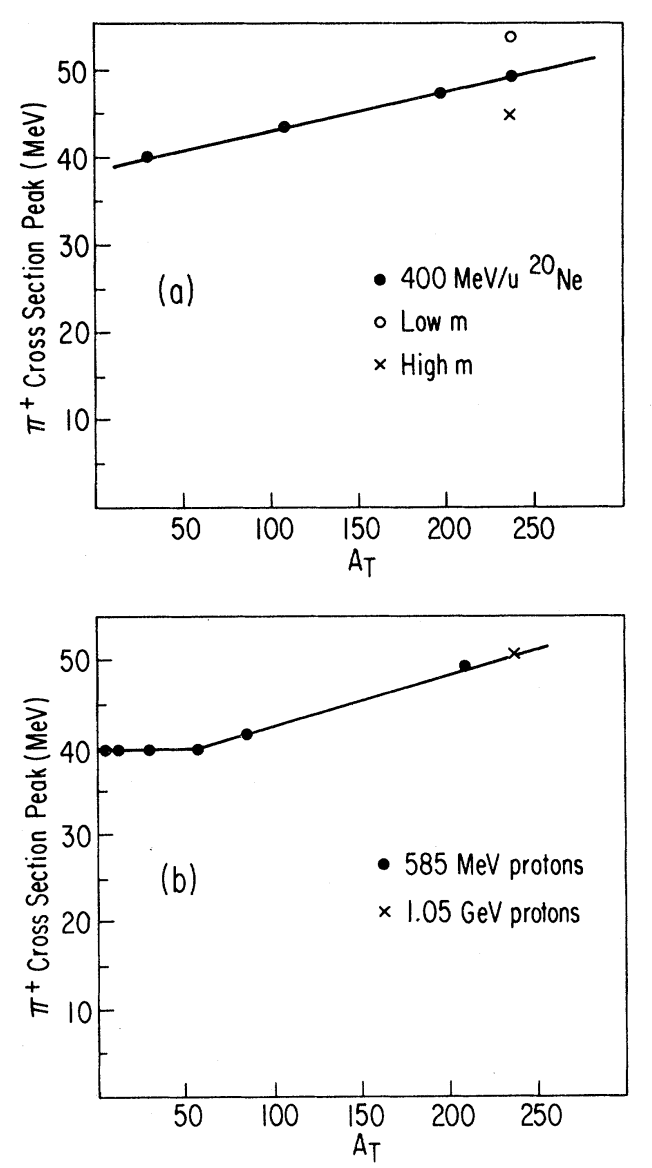

FIG. 11. The laboratory pion energy corresponding to cross section maxima for (a) $130^{\circ}$ and $150^{\circ}$ data from $400 \mathrm{MeV} / \mathrm{u}{ }^{20} \mathrm{Ne}$ as a function of target mass, $A_{T}$ (solid points). Results for $1.05 \mathrm{GeV} / \mathrm{u}{ }^{20} \mathrm{Ne}+{ }^{238} \mathrm{U}$ fall on the same line, with low multiplicity $(O)$ and high multiplicity $(X)$ selections, respectively; (b) $135^{\circ}$ for $585 \mathrm{MeV} p$ (Ref. 19) and $1.05 \mathrm{GeV} p$.

data between low and high multiplicity restrictions are small, but from the discussion in subsection B of this section, for such light systems, the use of multiplicity selection is expected to produce only very crude impact parameter restrictions due to statistical fluctuations.

Figure 13(a) shows data for $400 \mathrm{MeV} / \mathrm{u}$ ${ }^{40} \mathrm{Ar}+{ }^{40} \mathrm{Ca}$, which have been reflected through $\left(y_{p}+y_{T}\right) / 2,90^{\circ}$ c.m., assuming the target and projectile are identical. Unfortunately, statistical uncertainties in this particular set of data are too large to allow significant impact parameter restrictions to be made. An increase of the laboratory bombarding energy for ${ }^{40} \mathrm{Ar}+{ }^{40} \mathrm{Ca}$ results in a significant change in the pion inclusive spectra, as shown in Fig. 13(b). The $\pi^{+}$emission pattern is dominated 

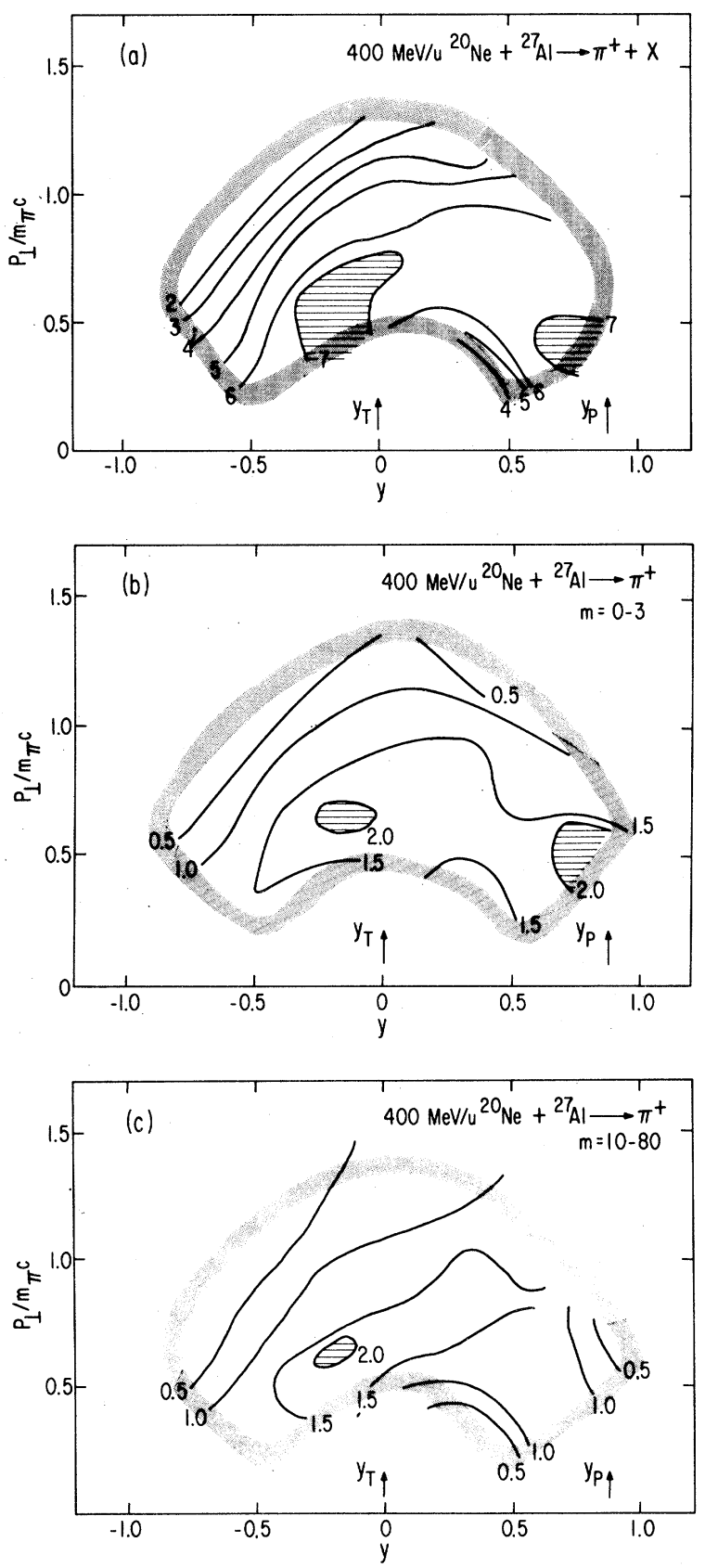

FIG. 12. Same as Fig. 10 except the target-projectile combination is $400 \mathrm{MeV} / \mathrm{u}{ }^{20} \mathrm{Ne}+{ }^{27} \mathrm{Al}, f=10^{-4}$.

by a cross section maximum at midrapidity $\left(90^{\circ}\right.$ c.m.) and $p_{\perp} \approx 0.4 m_{\pi}\left(E_{\pi}+\sim 15-20 \mathrm{MeV}\right)$, instead of the $0^{\circ}, 180^{\circ}$ emission expected in isobar decay seen in $p+p \rightarrow \pi^{+}+X$ reactions. ${ }^{15,19,21}$ It may be compared with the $730 \mathrm{MeV} p+p$ data of Cochran et al. ${ }^{15}$ for $\pi^{+}$production in Fig. 13(c). In Fig. 14 pion angular distributions are presented for the 1.05 $\mathrm{GeV} / \mathrm{u}{ }^{40} \mathrm{Ar}+{ }^{40} \mathrm{Ca}$ reaction in the center-of-mass
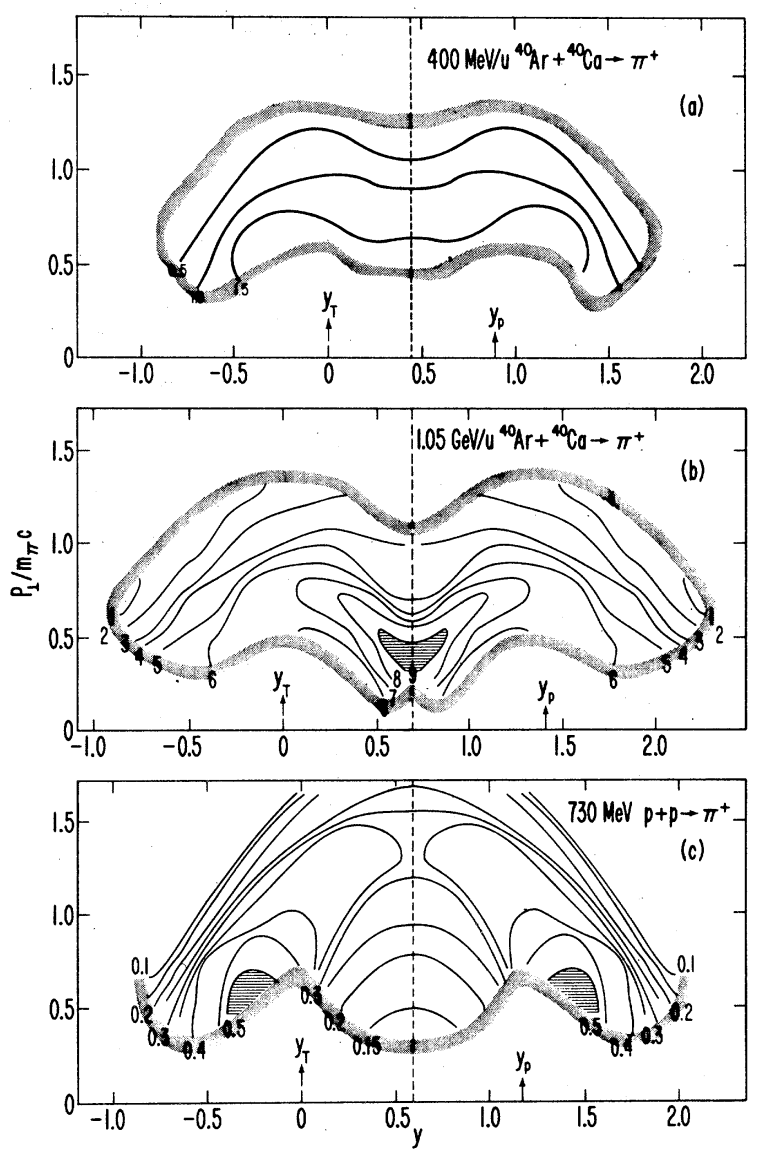

FIG. 13. (a) Same as the rapidity plot in Fig. 10(a) except the target-projectile combination is $400 \mathrm{MeV} / \mathrm{u}$ ${ }^{40} \mathrm{Ar}+{ }^{40} \mathrm{Ca}, f=10^{-3}$. The data have been reflected through midrapidity $\left(y_{P}+y_{T}\right) / 2$, shown by the dashed vertical line. (b) and (c) are the same as the rapidity plot of Fig. 10 except the target-projectile combination is $1.05 \mathrm{GeV} / \mathrm{u}{ }^{40} \mathrm{Ar}+{ }^{40} \mathrm{Ca}$ and $730 \mathrm{MeV} p+p$, $f=10^{-3}$, respectively.

coordinate system, every $50 \mathrm{MeV} / c$ of c.m. pion momentum from 50 to $300 \mathrm{MeV} / c$. Data for $\pi^{+}$ production in the $800 \mathrm{MeV} / \mathrm{u}^{40} \mathrm{Ar}+\mathrm{KCl}$ reaction ${ }^{14}$ are used to supplement the $\pi^{+}$data taken in the present study at high momentum around $90^{\circ} \mathrm{c} . \mathrm{m}$. and are renormalized to the data taken in the present study by multiplying by a factor of 1.8 . For the $p+p$ reaction, the angular distribution is forward-backward peaked for all $p_{\pi^{+}}$, and the maximum yield occurs around $200 \mathrm{MeV} / c$, as predicted by the isobar model. The $\pi^{+}$angular distribution for the $1.05 \mathrm{GeV} / \mathrm{u}{ }^{40} \mathrm{Ar}+{ }^{40} \mathrm{Ca}$ reaction is also forward-backward peaked near resonance energies, like the theoretically predicted $w(\theta) \approx 3 \cos ^{2} \theta+1$ behavior in $p+p$ reactions shown by the dashed line 


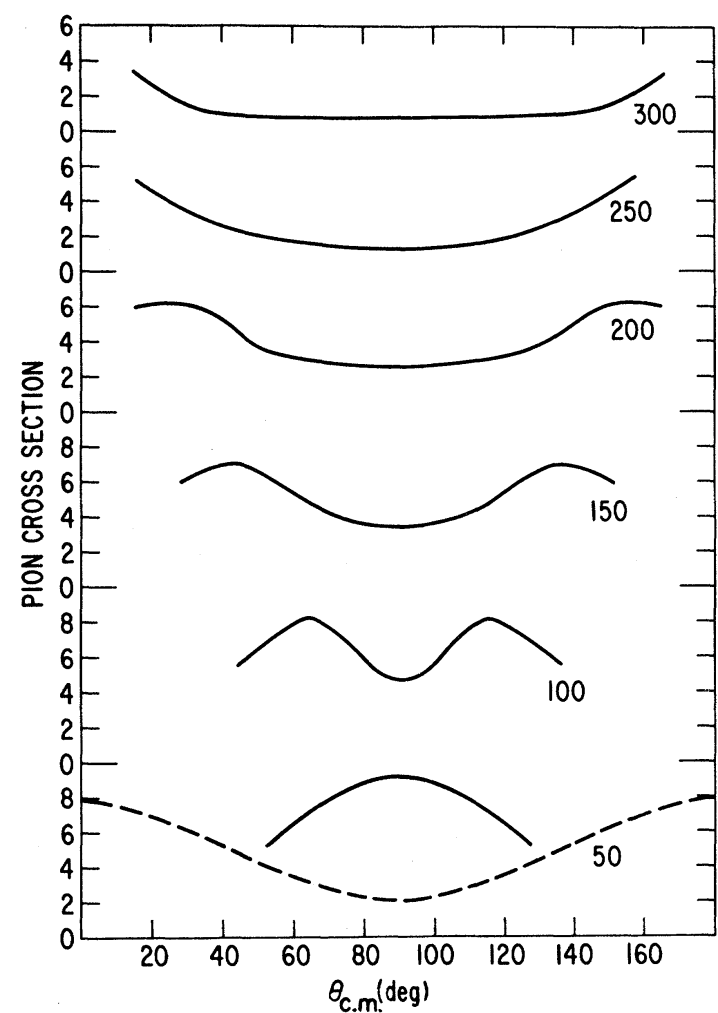

FIG. 14. Center-of-mass angular distributions every $50 \mathrm{MeV} / c$ of pion momentum for the $1.05 \mathrm{GeV} / \mathrm{u}$ ${ }^{40} \mathrm{Ar}+{ }^{40} \mathrm{Ca} \rightarrow \pi^{+}+X$ reaction. The dashed line shows the shape predicted by the isobar model in $p+p$ reactions near resonance. Invariant cross section $1 / p\left(d^{2} \sigma / d \Omega d E\right)$ is in units of $\left(\mathrm{mb} c^{3} / \mathrm{MeV}^{2} \mathrm{sr}\right) \times 10^{-1}$.

in Fig. 14. For the ${ }^{40} \mathrm{Ar}+{ }^{40} \mathrm{Ca}$ reaction shown in Fig. 14, the angular distributions for lower momenta have maxima at intermediate angles, and finally for $p_{\pi^{+}} \approx 50 \mathrm{MeV} / c$, the yield is large and peaks at $90^{\circ}$ c.m.

Rapidity plots of two of the calculations discussed earlier provide some insight for the ${ }^{40} \mathrm{Ar}+{ }^{40} \mathrm{Ca}$ reaction. Results of cascade I and the firestreak model ${ }^{5}$ are shown in Figs. 15(a) and (b), respectively. The emission pattern generated by the intranuclear cascade code looks much like the $p+p$ data, since it uses the isobar model for production and absorption. The firestreak calculation produces a midrapidity bump in the $\pi^{+}$yield of the same spirit as the data.

Multiplicity-selected data for the $1.05 \mathrm{GeV} / \mathrm{u}$ ${ }^{40} \mathrm{Ar}+{ }^{40} \mathrm{Ca}$ reaction are shown in Figs. 16(a) and (b) for peripheral and central collisions, respectively. Here the data are not reflected about $90^{\circ} \mathrm{c.m}$. The backward-going ridge which is characteristic of isobar decay is present for low multiplicities, but is
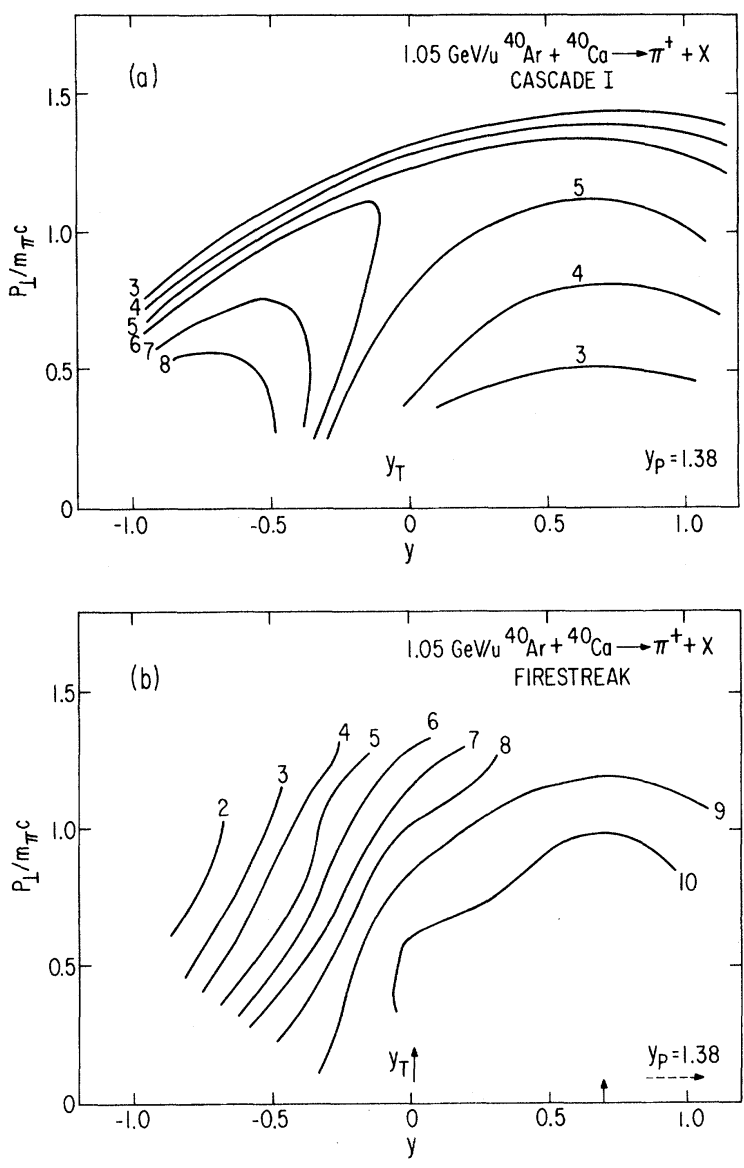

FIG. 15. Rapidity plots of calculations done with (a) the intranuclear cascade model (Ref. 1) and (b) the firestreak model (Ref. 5), $f=10^{-3}$.

only comparable in strength to the midrapidity bump. For central collisions, the structure at $90^{\circ}$ c.m. is clearly dominant, but as shown elsewhere ${ }^{6}$ the shapes of the spectra at midrapidity are insensitive to the impact parameter.

The $\pi^{+}$emission patterns for relatively low energy light projectiles, $p$ and ${ }^{4} \mathrm{He}$, on heavy targets is dominated by a back-angle ridge, as discussed earlier for the ${ }^{4} \mathrm{He}+{ }^{27} \mathrm{Al}$ reaction. This can be seen in Fig. 17 for $250 \mathrm{MeV} / \mathrm{u}{ }^{20} \mathrm{Ne}+{ }^{238} \mathrm{U}$, as well as for $1.05 \mathrm{GeV} / \mathrm{u}$ proton- and ${ }^{4} \mathrm{He}$-induced reactions on ${ }^{238} \mathrm{U}$ shown in Figs. 18(a) and (b), respectively. Data for $400 \mathrm{MeV} / \mathrm{u}{ }^{20} \mathrm{Ne}+{ }^{238} \mathrm{U}$ are very similar. The Coulomb-isobar ridge also occurs for 1.05 $\mathrm{GeV} / \mathrm{u}{ }^{20} \mathrm{Ne}+{ }^{238} \mathrm{U}$ [Fig. 19(a)], but an additional island of yield occurs at midrapidity, apparently from the $N-N$ frame. Figure 19(c) shows that this forward-angle maximum is a property of central collisions. The $\pi^{+}$-inclusive data of Nakai et al. ${ }^{31}$ show a similar but weaker structure for $800 \mathrm{MeV} / \mathrm{u}$ 

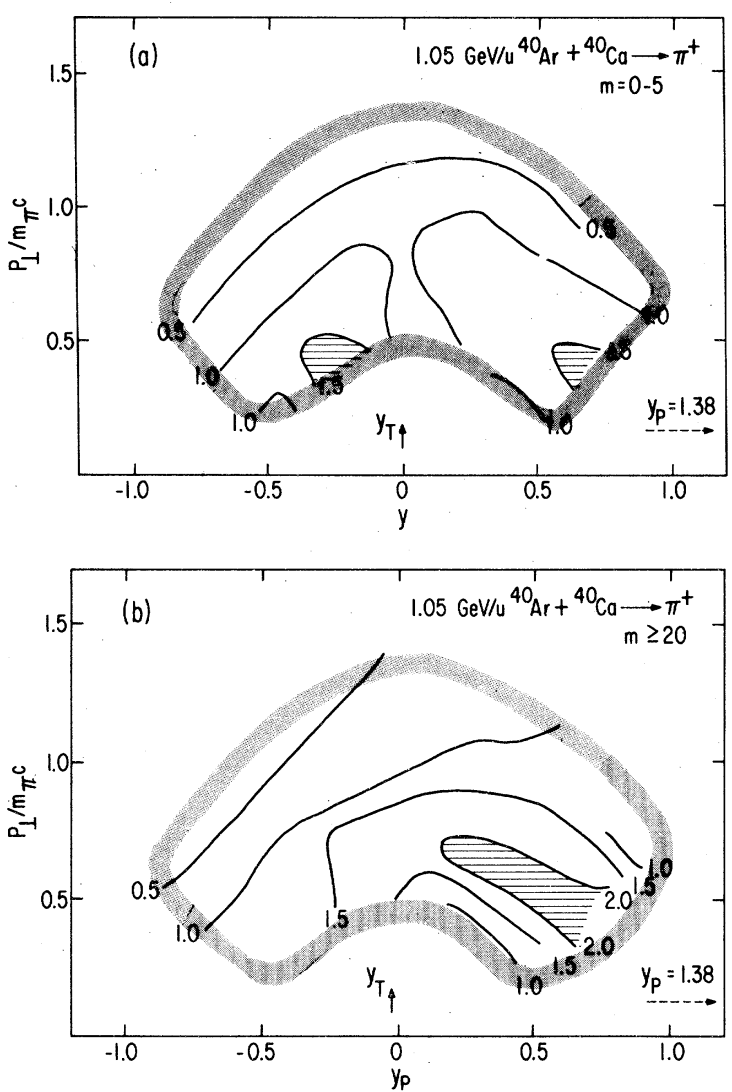

FIG. 16. Rapidity plots of $\pi^{+}$data for the 1.05 $\mathrm{GeV} / \mathrm{u}{ }^{40} \mathrm{Ar}+{ }^{40} \mathrm{Ca}$ reaction multiplicity gated with (a) $0-5$ charged particles and (b) $20-80$ charged particles, $f=10^{-3}$.

${ }^{20} \mathrm{Ne}+{ }^{208} \mathrm{~Pb}$. A significant change occurs for the ${ }^{20} \mathrm{Ne}+{ }^{238} \mathrm{U}$ reaction at $2.1 \mathrm{GeV} / \mathrm{u}$ (Fig. 20), with the back-angle ridge being absent. The telescope used here is inadequate for measuring much of the

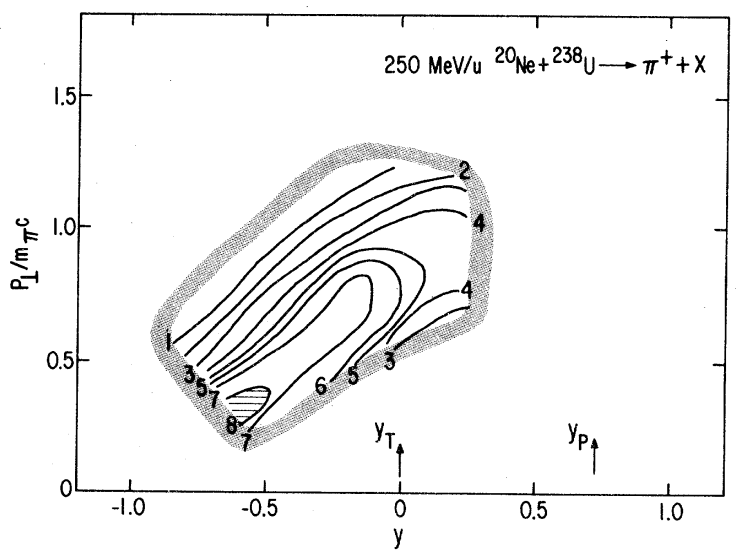

FIG. 17. Rapidity plot of $\pi^{+}$-inclusive data from the $250 \mathrm{MeV} / \mathrm{u}{ }^{20} \mathrm{Ne}+{ }^{238} \mathrm{U}$ reaction, $f=10^{-4}$.
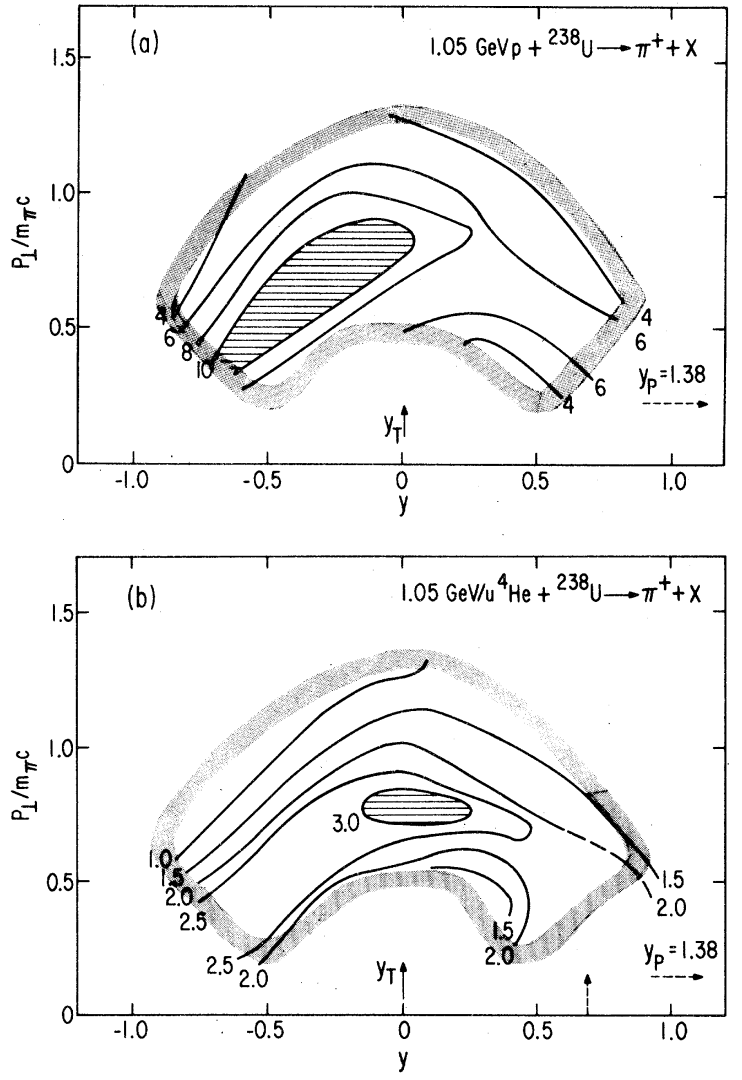

FIG. 18. Rapidity plots of $\pi^{+}$-inclusive data from (a) $1.05 \mathrm{GeV} p+{ }^{238} \mathrm{U}$ reaction, $f=10^{-4}$, and (b) 1.05 $\mathrm{GeV} / \mathrm{u}{ }^{4} \mathrm{He}+{ }^{238} \mathrm{U}$ reaction, $f=10^{-3}$.

pion spectra produced at this high bombarding energy. The multiplicity gating shows large differences (factors of 5) between low and high selections in some areas. The more central impact parameters show a large enhancement at the highest $p_{\perp}$ values at $90^{\circ}$ in the $N-N$ center of mass, but more complete information is needed to draw any definite conclusions. The change in the emission pattern of the $\pi^{+}$-inclusive data compared to lower bombarding energies can be qualitatively understood in terms of the isobar model, with multiple pion production in a single $N-N$ collision. From 1.0 to $2.3 \mathrm{GeV}$ proton-induced $\pi^{+}$production on hydrogen and ${ }^{9} \mathrm{Be}$ targets ${ }^{27}$ it is known that the characteristic $\pi^{+}$ energy from isobar decay of $\approx 150 \mathrm{MeV}(p \approx 200$ $\mathrm{MeV} / c$ ) in the $N-N$ center of mass is maintained in multiple pion production. Thus in the ${ }^{20} \mathrm{Ne}+{ }^{238} \mathrm{U}$ reaction at $2.1 \mathrm{GeV} / \mathrm{u}$, the $N-N$ frame is moving at a high laboratory velocity, such that the $\pi^{+}$emitted toward back angles in the c.m. are still moving forward in the laboratory with low velocity. This, along with the Coulomb repulsion from the urani- 

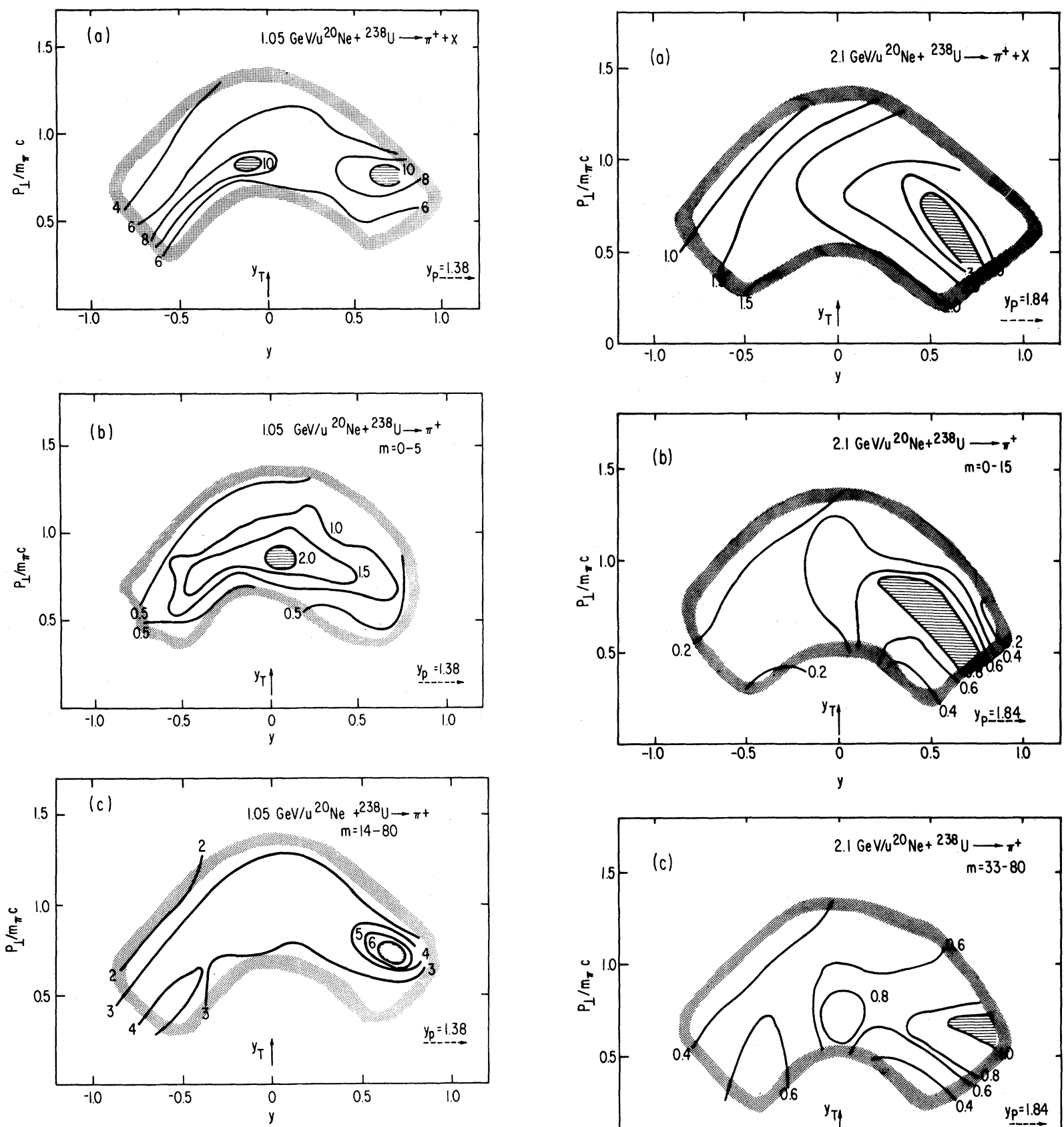

FIG. 19. Rapidity plots of $\pi^{+}$data for the 1.05 $\mathrm{GeV} / \mathrm{u}{ }^{20} \mathrm{Ne}+{ }^{238} \mathrm{U}$ reaction for (a) single-particle inclusive, (b) low multiplicity gating, and (c) high multiplicity gating, $f=10^{-3}$.

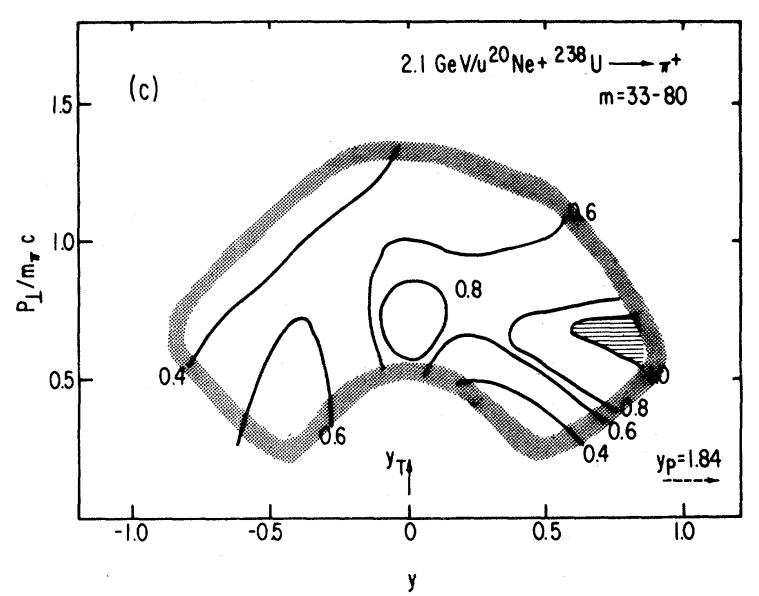

FIG. 20. Rapidity plots of $\pi^{+}$data for the 2.1 $\mathrm{GeV} / \mathrm{u}{ }^{20} \mathrm{Ne}+{ }^{238} \mathrm{U}$ reaction for (a) single-particle inclusive, (b) low multiplicity, and (c) high multiplicity, $f=10^{-2}$.

umlike spectator fragment (nearly at rest in the laboratory system) results in a forward-going ridge. The $1.05 \mathrm{GeV} / \mathrm{u}{ }^{40} \mathrm{Ar}+{ }^{238} \mathrm{U} \rightarrow \pi^{+}+X$ reaction has properties of the ${ }^{20} \mathrm{Ne}+{ }^{238} \mathrm{U}$ reaction at 1.05 and $2.1 \mathrm{GeV} / \mathrm{u}$, as can be seen in Fig. 21. A back-angle

ridge and an enhancement at midrapidity occur in the more central impact parameter restricted data, but the structure at midrapidity is not as prominent as in the ${ }^{20} \mathrm{Ne}+{ }^{238} \mathrm{U}$ reaction. 

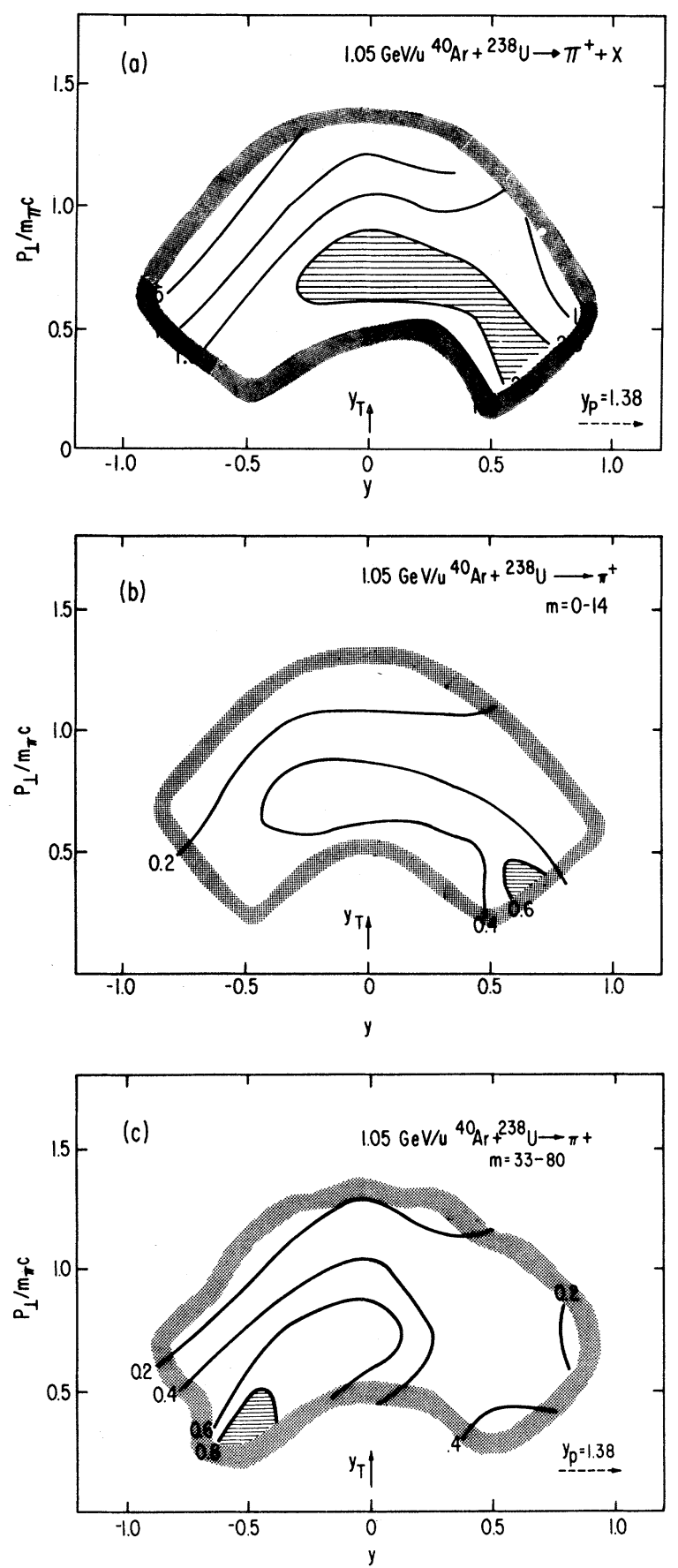

FIG. 21. Rapidity plots of $\pi^{+}$data for the 1.05 $\mathrm{GeV} / \mathrm{u}{ }^{40} \mathrm{Ar}+{ }^{238} \mathrm{U}$ reaction for (a) single-particle inclusive, (b) low multiplicity, and (c) high multiplicity, $f=1$.

\section{DISCUSSION}

In the preceding section the experimental results were presented and some qualitative explanations were given for effects seen in the data. In this section results are discussed in terms of scaling of the pion yields with respect to target and projectile masses. The effect of the Coulomb force on pion emission patterns is investigated with a simple trajectory calculation.

\section{A. Scaling with target and projectile masses}

Comparisons of $\pi^{+}$yields from proton plus nucleus reactions ${ }^{16-18}$ led to the conclusion that pion absorption was the dominant factor controlling the observed $Z_{T}^{1 / 3}$ or $A_{T}^{1 / 3}$ dependence. A comparison is shown in Fig. 22 for $\pi^{+}$emission at $50^{\circ}$ in the laboratory, approximately $90^{\circ}$ in the $N-N$ center of mass over the pion energy range measured here, for $400 \mathrm{MeV} / \mathrm{u}^{20} \mathrm{Ne}$ on several targets. The scaling parameters that might apply to the present data are not necessarily expected $a$ priori to be the usual surface area-dependent quantities. Since the bombarding energy is well below resonance in the $N-N$ frame, the low energy pions produced are not expected to be strongly absorbed. In fact, a $Z^{1 / 3}$ or $A^{1 / 3}$ dependence gives a poor representation of the $50^{\circ}$ data. Similarly, the $90^{\circ}$ laboratory data, which may be more applicable to the target mass dependence, have a virtually identical behavior, falling be-

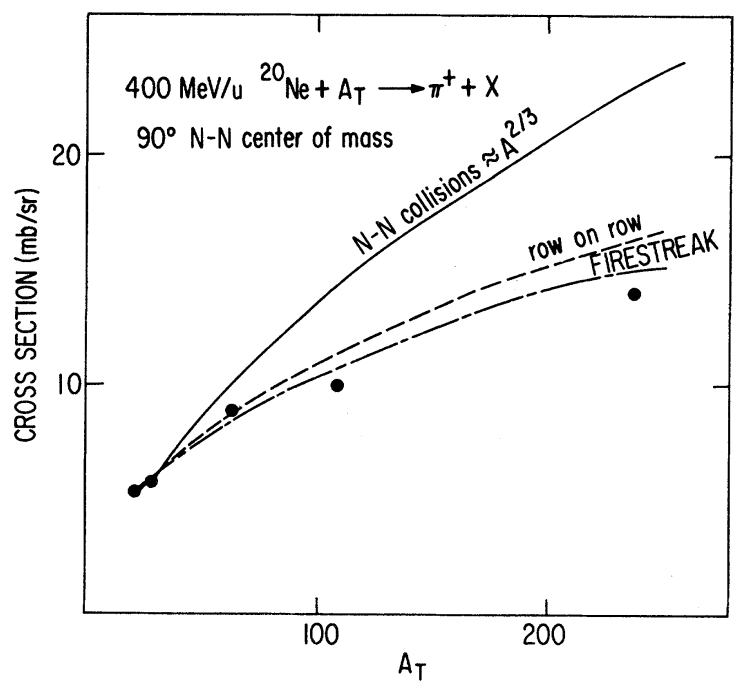

FIG. 22. Energy integrated cross sections, from 15-95 MeV for $\pi^{+}$emitted at $\theta_{\mathrm{lab}}=50^{\circ}$ produced with $400 \mathrm{MeV} / \mathrm{u}{ }^{20} \mathrm{Ne}$ projectiles on targets of mass $A_{T}$. Data for ${ }^{27} \mathrm{Al}$, ${ }^{\text {nat }} \mathrm{Ag},{ }^{197} \mathrm{Au}$, and ${ }^{238} \mathrm{U}$ are from the present work. Points for $\mathrm{NaF}$ and ${ }^{\text {nat }} \mathrm{Cu}$ are from Ref. 31. Calculations have been renormalized to ${ }^{27} \mathrm{Al}$. 
tween $A^{1 / 3}$ and $A^{2 / 3}$, the latter dependence expected in an $N-N$ first-collision model with no absorption. One may conclude that, while pion absorption is not dominant, it must be taken into consideration, as is done for the results shown in Fig. 22 in the linear cascade (row on row) model, ${ }^{38}$ and in the firestreak calculation through the assumption of chemical equilibrium in the participant region. While the target dependence of the cross sections is approximately correct, the absolute values of the pion cross sections predicted by the firestreak model $^{5}$ are approximately a factor of 2 above the measured values.

Well above delta resonance, for $1.05 \mathrm{GeV} / \mathrm{u}{ }^{40} \mathrm{Ar}$ on targets of ${ }^{40} \mathrm{Ca}$ and ${ }^{238} \mathrm{U}$, the steepness of the $A_{T}$ dependence increases in the target-rapidity region, nearly as $A_{T}{ }^{2 / 3}$, even further from the expected $Z_{T}^{1 / 3}$ behavior. At face value this result may be interpreted in terms of an increasing importance of $\pi^{+}$production through an individual $N-N$ collision mechanism as shown by the upper curve in Fig. 22. The validity of considering heavy ion collisions as a superposition of independent $N-N$ collisions may be tested in some detail here by comparing the results of $1.05 \mathrm{GeV} / \mathrm{u} p,{ }^{4} \mathrm{He},{ }^{20} \mathrm{Ne}$, and ${ }^{40} \mathrm{Ar}$ production of $\pi^{+}$on a ${ }^{238} \mathrm{U}$ target. To a good approximation, the $\pi^{+}$cross sections are expected to scale with the number of incident protons in the respective projectiles, with the relatively large target nucleus dominating the emission patterns in the target-rapidity region. Some success has been claimed ${ }^{12}$ for a similar treatment by using $\pi^{+}$and $\pi^{-}$production cross sections for proton plus nucleus reactions to calculate ${ }^{20} \mathrm{Ne}$-induced results. In Fig. 23, cross section ratios integrated over the energy range measured are presented as a function of laboratory angle. Because there is a minimum of transformations and most systematic effects cancel, these ratios are very accurate. In Fig. 23(a) one can see that, even in a comparison of ${ }^{4} \mathrm{He}$ - and $p$-induced $\pi^{+}$production, there is a significant deviation from the results expected in an independent $N-N$ collision assumption, represented by the dashed line. The isotropy of the calculated cross section represented by the dashed line reflects the expected importance of the heavy ${ }^{238} \mathrm{U}$ target for absorption and scattering. The absolute value was calculated from measured $p$ plus nucleus $\pi^{+}$and $\pi^{-}$production ${ }^{15}$ and may be understood with the isobar model. The $p+p$ reaction is the most important contributor for $\pi^{+}$, with the $p+n$ and secondary charge exchange reactions (since $N \neq Z$ ) leading to a $50 \%$ increase over scaling by the number of projectile protons. In parts (b)

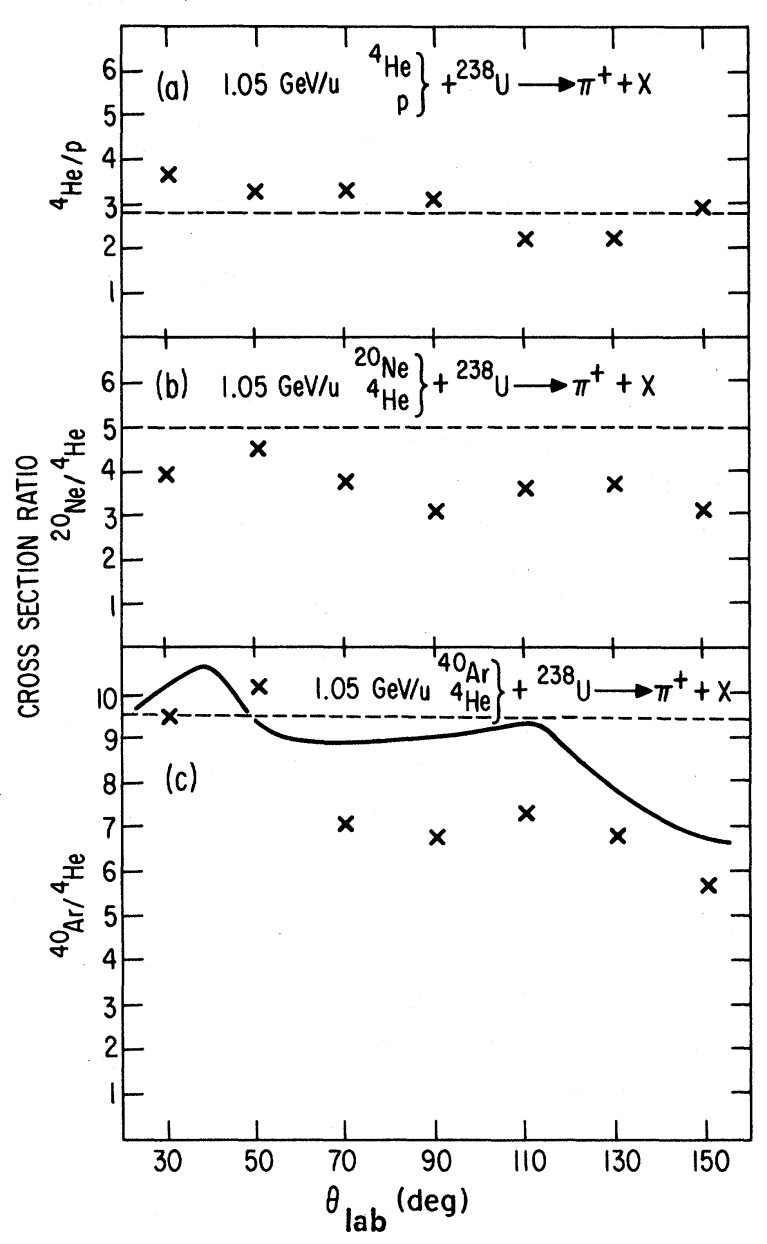

FIG. 23. Ratios of energy integrated cross sections $(15-95 \mathrm{MeV})$ as a function of laboratory angle for $\pi^{+}$ produced by $p,{ }^{4} \mathrm{He},{ }^{20} \mathrm{Ne}$, and ${ }^{40} \mathrm{Ar}$ at $1.05 \mathrm{GeV} / \mathrm{u}$ on a ${ }^{238} \mathrm{U}$ target. Dashed lines represent predictions of scaling from an isobar model. The solid curve in the bottom figure for ${ }^{40} \mathrm{Ar} /{ }^{4} \mathrm{He}$ presents the results of an intranuclear cascade calculation (cascade II) (Ref. 2).

and (c) of Fig. 23, a comparison of ${ }^{20} \mathrm{Ne}$ - and ${ }^{40} \mathrm{Ar}$ induced $\pi^{+}$production with the ${ }^{4} \mathrm{He}$ pion production shows an additional effect over the ${ }^{4} \mathrm{He} / p$ effect, leading to a lower pion yield than might be expected from independent $N-N$ collisions. In Fig. 23(c), the solid line represents the prediction of the cascade II calculation of Yariv and Fraenkel, ${ }^{2}$ believed to be one of the best calculations for pion production. Although the model works well at extreme forward and backward angles, there is a significant discrepancy at midangles (representing most of the production cross section), with the data being lower by approximately $25 \%$. While seemingly a small difference, actually it points to a serious shortcoming, since it is in scaling up from ${ }^{4} \mathrm{He}$ 
to ${ }^{40} \mathrm{Ar}$.

The low ${ }^{40} \mathrm{Ar} /{ }^{4} \mathrm{He}$ cross section ratio is more significant than the differences between the measured cross sections and the results of the calculations found for the ${ }^{4} \mathrm{He}+{ }^{238} \mathrm{U}$ and ${ }^{40} \mathrm{Ar}+{ }^{238} \mathrm{U}$ reactions shown in Figs. 6(b) and (c), respectively, which could be attributed to inaccuracies in the $N-N$ cross sections, for instance. From the effect seen for the ${ }^{40} \mathrm{Ar}+{ }^{40} \mathrm{Ca} \rightarrow \pi^{+}+X$ reaction in the comparison of the cascade I and cascade II calculations, it is tempting to attribute the present discrepancy to inadequate accounting of the cascade-cascade interactions in cascade II. Decreased pion yields could be obtained through a $N+\Delta \rightarrow N+N$ process, which should be important in the ${ }^{40} \mathrm{Ar}+{ }^{238} \mathrm{U}$ reaction. Detailed-balance considerations would seem to preclude this explanation, except for the restriction in cascade II that two cascade particles cannot interact more than once. A more "exotic" explanation, as given by Cugnon et al. ${ }^{39}$ deals with the chemical potential felt by the pions upon leaving the interaction region. Since there is an attractive potential between the pion and the nucleus, the pion yield would be reduced. Such an effect would seem to be more prominent in a well-defined large "fireball" for ${ }^{40} \mathrm{Ar}+{ }^{238} \mathrm{U}$ compared to the ${ }^{4} \mathrm{He}+{ }^{238} \mathrm{U}$ system.

\section{B. Coulomb trajectory calculations}

Coulomb effects have long been observed in pion spectra for proton plus nucleus reactions ${ }^{17,18}$ as demonstrated here in Fig. 11(b). In heavy ioninduced pion production, the first unambiguous demonstration of Coulomb final state interactions was the observation of Benenson et al ${ }^{40}$ of a sharp peak in $\pi^{+} / \pi^{-}$ratios near zero degrees at projectile velocities. Libbrecht and Koonin, ${ }^{34}$ and later Cugnon et al. ${ }^{41}$ and Gyulassy et al. ${ }^{42}$ have shown, with proper choices of parameters, that a considerable Coulomb focusing effect can be generated in calculations. This could explain the broad structures near $90^{\circ}$ c.m. seen for $\pi^{+}$for $1.05 \mathrm{GeV} / \mathrm{u}$ ${ }^{40} \mathrm{Ar}+{ }^{40} \mathrm{Ca}$ in the present work and in the 800 $\mathrm{MeV} / \mathrm{u}{ }^{20} \mathrm{Ne}+\mathrm{NaF}$ system. ${ }^{31}$ The Coulomb field produced by moving projectile and target remnants tends to focus $\pi^{+}$toward $\theta_{\text {c.m. }}=90^{\circ}$, and the Coulomb repulsion from a fireball at rest in the center of mass can lead to the formation of a peak in the $\pi^{+}$yield at $p_{\perp} \neq 0$. While seemingly able to account for broad structures in the above two systems, several questions have remained unanswered which cast doubt on an interpretation based upon
Coulomb effects. Of particular note is the disappearance of the $90^{\circ}$-enhanced cross sections, like that shown in Fig. 13(b) and Fig. $14(p=50 \mathrm{MeV} / c)$ when the bombarding $E / A$ is lowered to $400 \mathrm{MeV}$, as shown on Fig. 13(a). The occurrence of the midrapidity structure at somewhat higher values of $p_{\perp}$ for ${ }^{20} \mathrm{Ne}+\mathrm{NaF}$ (Ref. 31), as compared to the higher $Z{ }^{40} \mathrm{Ar}+{ }^{40} \mathrm{Ca}$, remains unexplained. Also with the observation of similar structures for $\pi^{+}$ from central collisions of ${ }^{20} \mathrm{Ne}+{ }^{238} \mathrm{U}$ at $1.05 \mathrm{GeV} / \mathrm{u}$ and possibly at $2.1 \mathrm{GeV} / \mathrm{u}$ (but not in proton- or ${ }^{4} \mathrm{He}$-induced reactions) it seems necessary to perform Coulomb trajectory calculations that address all of the data.

Following the method of Libbrecht and Koonin, ${ }^{34}$ calculations were performed using numerical solution of the classical equations of motion in the time dependent field of moving charges. The procedure outlined by Fraenkel et $a l .{ }^{43}$ was used for three or four point charges, and generalized to be fully relativistic. Later, the effects of the finite nucleus size and time of pion emission (early or late in the collision) were investigated.

The results of previous calculations, with appreciable focusing toward $90^{\circ}$ c.m., could be qualitatively reproduced by allowing $\pi^{+}$emission to occur at the time of maximum overlap in the ${ }^{40} \mathrm{Ar}+{ }^{40} \mathrm{Ca}$ system at $1.05 \mathrm{GeV} / \mathrm{u}$. Impact parameter weighting was made according to the participant-spectator model. A possible explanation for the difference in $\pi^{+}$-emission patterns observed at $400 \mathrm{MeV} / \mathrm{u}$ and $1.05 \mathrm{GeV} / \mathrm{u}$ is demonstrated in Fig. 24. As an alternative to using a fireball model to generate the undistorted pion source function as has been done previously, ${ }^{34}$ complete transparency was assumed with no charge at rest in the center of mass. To obtain a peak at $p_{\perp} \neq 0$ at $90^{\circ} \mathrm{c.m}$., it is necessary for the undistorted pion spectrum to have a peak at nonzero momentum. ${ }^{34}$ In Fig. 24(a) it was assumed that the undistorted emission pattern was that of isobar decay, with an angular distribution given by $w(\theta) \sim 3 \cos ^{2} \theta+1$, and with a pion momentum range of $170-220 \mathrm{MeV} / c$ for a nonzero cross section. This is a pattern similar to that observed in the $p+p \rightarrow \pi^{+}+X$ reaction, as shown in Fig. 13(c). The calculated emission pattern shown in Fig. 24(a) has several features in common with the experimental data shown in Fig. 13(b), including a broad bump of yield at $p_{1} \approx 0.5 m_{\pi} c$ and a winged structure extending away from $90^{\circ}$ c.m. Figure 24(b) presents the calculated rapidity plot for $400 \mathrm{MeV} / \mathrm{u}$ ${ }^{40} \mathrm{Ar}+{ }^{40} \mathrm{Ca}$, with the same conditions, except the undistorted emission pattern has been changed to a 


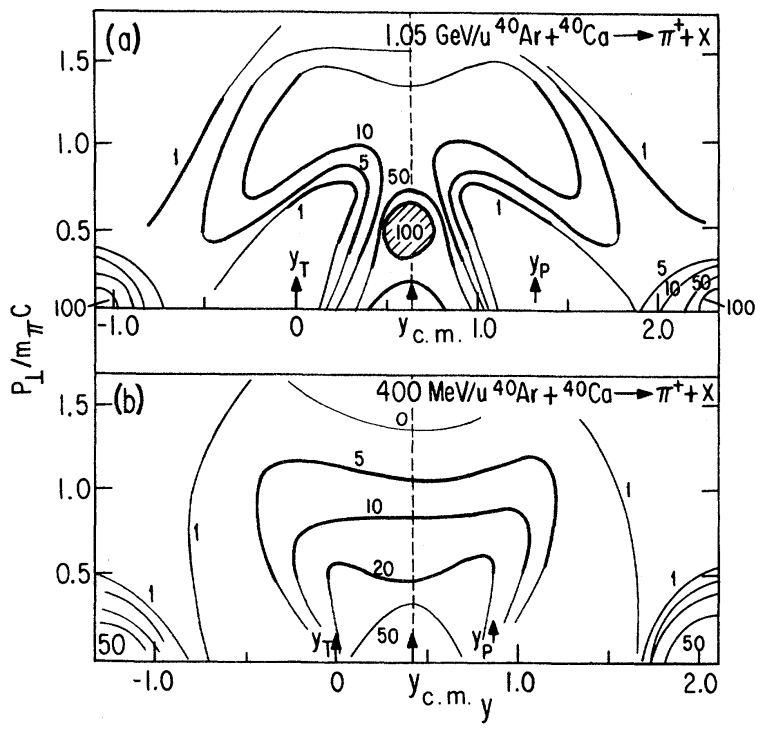

FIG. 24. Rapidity plots for $\pi^{+}$generated by Coulomb trajectory calculations for ${ }^{40} \mathrm{Ar}+{ }^{40} \mathrm{Ca}$ which assume complete transparency of the target and projectile and $\pi^{+}$emission at the time of maximum overlap of the nuclides. Undistorted source functions are (a) isobar emission pattern, $w(\theta) \sim 3 \cos ^{2} \theta+1, \quad 170 \leq P_{\pi} \leq 220$ $\mathrm{MeV} / c$, and $E / A=1.05 \mathrm{GeV}$; (b) a uniform distribution, $w(\theta)=1, \quad 0 \leq P_{\pi} \leq 200 \mathrm{MeV} / c$, and $E / A=400$ $\mathrm{MeV}$.

uniform distribution over the range $0 \leq p \leq 200$ $\mathrm{MeV} / c$. The rationale for this change being, for bombarding energies far below the 3-3 resonance energy, the isobar emission pattern is smeared by the Fermi motion as discussed earlier. A dramatic difference is thus accounted for at two bombarding energies, and can be attributed to:

(a) the difference in the ion-ion velocities, being slower than the bulk of the pions for $400 \mathrm{MeV} / \mathrm{u}$ and faster than the isobar-associated $\pi^{+}$at 1.05 $\mathrm{GeV} / \mathrm{u}$; and

(b) a change in the undistorted source functions, involving the classic isobar production and decay features.

This rather convenient explanation is questionable, however, due to delocalization effects, i.e., the pion wave function extends over a large spatial region for low pion energies, and will dilute considerably the focusing predicted by the classical model used here. Additional experimental data for $\pi^{+}$production using heavy targets also casts doubt on the Coulombeffect interpretation. In Fig. 19 for $1.05 \mathrm{GeV} / \mathrm{u}$ ${ }^{20} \mathrm{Ne}+{ }^{238} \mathrm{U}$, it can be seen that a midrapidity structure occurs, especially in central collisions. It should be noted that the cross section maximum occurs at the $N-N$ center of mass instead of the fireball frame for this asymmetric system. Results of the previously described Coulomb trajectory calculation yield results shown in Fig. 25, which are quite different from the experimental data of Fig. 19(a). As might be expected, the high $Z$ of the ${ }^{238} U$ target nucleus can produce a large distortion of the isobar pattern when pions are emitted in the early stages of the reaction. Besides the absence of a midrapidity maximum in the calculated emission pattern, also there is a large displacement of the Coulomb-isobar ridge at back-angles to $\left(y, p_{\perp}\right)$ values which are far greater than the experimental values. Much better agreement is obtained with the data at angles larger than $90^{\circ}$ by assuming that pions are emitted relatively late in the course of the reaction. This assumption neglects the "direct" pions ${ }^{39}$ and allows emission at a late stage of the reaction at final contact of the target and projectile nuclides, and only from the nuclear surface. The results of this calculation, again assuming complete transparency and an isobar-emission source function, are shown in Fig. 26. The position of the Coulomb-isobar ridge (near $Y_{T}$ ) is in good agreement with the experimental value. The Coulomb effects are much diminished, and also allow a focusing of the $\pi^{+}$toward the $N-N$ center of mass in this particular instance. As might be expected, however, no peak which follows the $N-N$ center of mass can be produced with a nucleus-nucleus Coulomb effect in other systems where this structure is observed, i.e., $0.8 \mathrm{GeV} / \mathrm{u}{ }^{20} \mathrm{Ne}+{ }^{208} \mathrm{~Pb},{ }^{31} 2.1 \mathrm{GeV} / \mathrm{u}{ }^{20} \mathrm{Ne}+{ }^{238} \mathrm{U}$, as well as the lighter systems. For the ${ }^{40} \mathrm{Ar}+{ }^{40} \mathrm{Ca}$ reaction, the calculated rapidity plot with the late emission assumption is shown in Fig. 27. The previously-obtained good agreement with the data

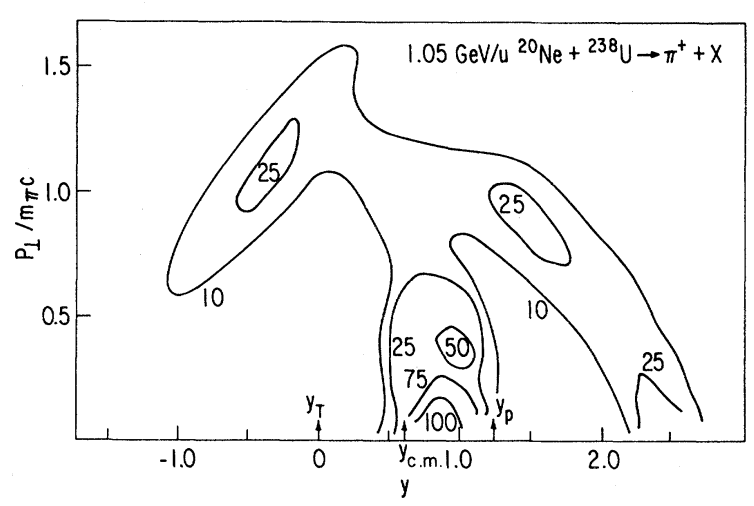

FIG. 25. Same as Fig. 24(a) except the targetprojectile combination is $1.05 \mathrm{GeV} / \mathrm{u}{ }^{20} \mathrm{Ne}+{ }^{238} \mathrm{U}$. 


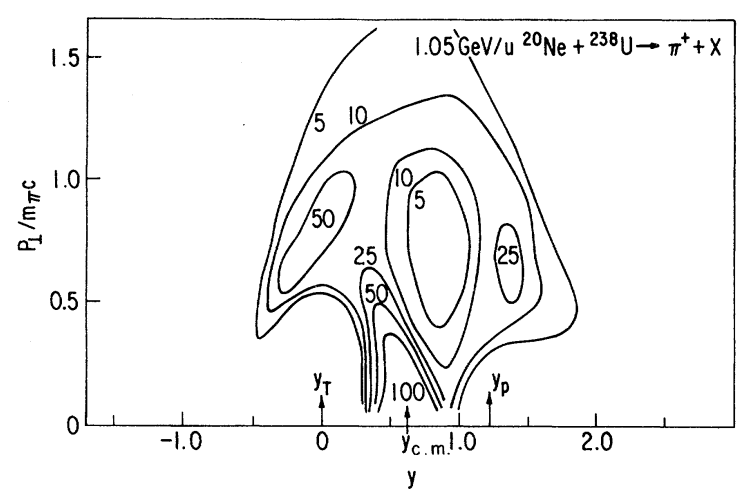

FIG. 26. Same as Fig. 25 except $\pi^{+}$are emitted later from the nuclear surface at the last stages of the reaction.

has been destroyed. The use of other source functions, i.e., a uniform distribution or a fireball model, also fails to provide a consistent explanation of the midrapidity maxima for all of the data. The assumption of late emission of the pions, or alternatively, of a large emission radius, seems to be the most reasonable one in accounting for the wellestablished Coulomb-effect at back angles. This suggests that the effect of the Coulomb field is minimal for the pion emission patterns near $\theta_{\text {c.m. }}=90^{\circ}$ in light systems such as ${ }^{20} \mathrm{Ne}+\mathrm{NaF}$ or ${ }^{40} \mathrm{Ar}+{ }^{40} \mathrm{Ca}$. Such an interpretation is consistent with recent $\pi^{+} / \pi^{-}$ratio measurements, ${ }^{44}$ which produce values close to unity for the ${ }^{40} \mathrm{Ar}+{ }^{40} \mathrm{Ca}$ reaction at $1.05 \mathrm{GeV} / \mathrm{u}$ in the region of midrapidity. In the calculation performed in Fig. 24 with early emission of pions, the $\pi^{+} / \pi^{-}$ratio undergoes variations of approximately a factor of 5-10. While there may be different sources of pions such as Cugnon's ${ }^{39}$ "direct" and "indirect" components, all of the calculations and comparisons performed here suggest strongly that Coulomb effects are of minimal importance for light systems. A modification of cascade calculations to include Coulomb effects would provide further insight. Specific explanations of a more positive sense for the midrapidity bumps, or enhanced yields at $90^{\circ} \mathrm{c} . \mathrm{m}$. observed around $1 \mathrm{GeV} / \mathrm{u}$, may point to more exotic phenomena ${ }^{45-47}$ which could allow late emission of low energy pions.

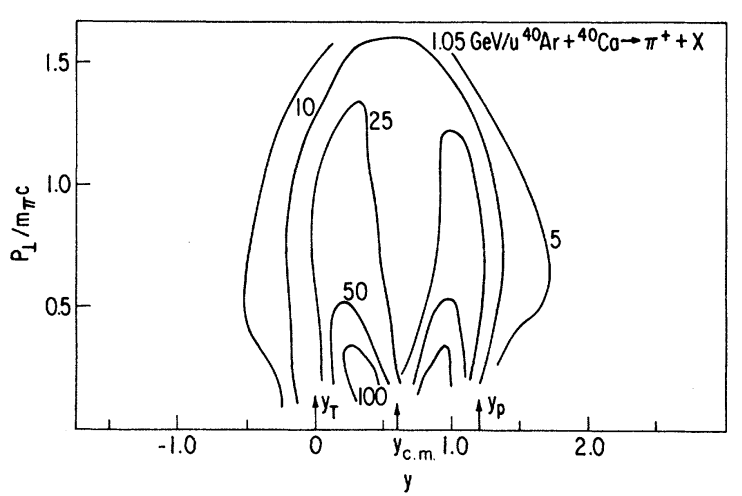

FIG. 27. Same as Fig. 26 except that target-projectile combination is $1.05 \mathrm{GeV} / \mathrm{u}{ }^{40} \mathrm{Ar}+{ }^{40} \mathrm{Ca}$.

\section{SUMMARY}

The systematics of $\pi^{+}$emission in heavy ion collisions has been investigated. Comparisons of the experimental results to several models have been made on two levels, the gross and the detailed properties. The simple models such as firestreak and rows on rows predict correctly the general trends in pion cross sections with respect to bombarding energies and target-projectile mass, but fail upon more quantitative considerations. The intranuclear cascade model gives better overall agreement, with some discrepancies appearing in careful comparisons of ${ }^{4} \mathrm{He}$ with heavier projectile-induced reactions.

A comparison to proton-nucleus production of pions has been made, with several features found to be in common with heavy ion-induced reactions. The apparent surface-production of $\pi^{+}$for heavy targets is similar, as well as the Coulomb-isobar ridge that occurs at backward angles for heavy targets. Enhanced yields of low energy pions near $90^{\circ}$ in the nucleon-nucleon center of mass seems to be a feature unique to heavy ion-induced reactions around $1 \mathrm{GeV} / \mathrm{u}$, a feature which is not readily explainable with models available at this time.

This work was performed under the auspices of the Office of High Energy and Nuclear Physics, Division of Nuclear Physics, U.S. Department of Energy.

\footnotetext{
(a)Present address: Texas A\&M University, College Station, TX 77843.

(b) Present address: Lawrence Berkeley Laboratory, Berkeley, CA 94720.
}

(c) Present address: Bell Laboratories, Reading, PA 19604.

(d) Present address: DPhN/ME, CEN de Saclay 91191 Gif sur Yvette Cedex, France. 
(e)Present address: IPN, BP1 91406 Orsay, France.

(f) Present address: Bell Laboratories, Murray Hill, NJ 07974.

(g) Present address: Schlumberger-Doll Research Center, Ridgefield, CT 06877.

(h) Present address: Institut des Sciences Nucléaires, 38026 Grenoble, France.

(i) Present address: Heavy Ion Laboratory, Michigan State University, East Lansing, MI 48824.

${ }^{1}$ Y. Yariv and Z. Fraenkel, Phys. Rev. C 20, 2227 (1979).

${ }^{2}$ Y. Yariv and Z. Fraenkel, Phys. Rev. C $\underline{24}, 488$ (1981).

3J. Gosset, H. H. Gutbrod, W. G. Meyer, A. M. Poskanzer, A. Sandoval, R. Stock, and G. D. Westfall, Phys. Rev. C 16, 629 (1977).

${ }^{4}$ W. D. Myers, Nucl. Phys. A296, 177 (1978).

5J. Gosset, J. I. Kapusta, and G. D. Westfall, Phys. Rev. C $\underline{18}, 844$ (1978).

${ }^{6} \mathrm{~K}$. L. Wolf, H. H. Gutbrod, W. G. Meyer, A. M. Poskanzer, A. Sandoval, R. Stock, J. Gosset, C. H. King, G. King, Nguyen Van Sen, and G. D. Westfall, Phys. Rev. Lett. 42, 1448 (1979).

${ }^{7}$ A. Sandoval, H. H. Gutbrod, W. G. Meyer, R. Stock, Ch. Lukner, A. M. Poskanzer, J. Gosset, J.-C. Jourdain, C. H. King, G. King, Nguyen Van Sen, G. D. Westfall, and K. L. Wolf, Phys. Rev. C $\underline{21}, 1321$ (1980).

${ }^{8}$ H. H. Gutbrod, M. R. Maier, H. G. Ritter, A. I. Warwick, F. Weik, H. Weiman, and K. L. Wolf, IEEE Trans. Nucl. Sci. NS-28, No. 1 (1981).

${ }^{9}$ K. A. Breuckner, R. Serber, and K. M. Watson, Phys. Rev. 84, 258 (1951).

${ }^{10}$ K. G. R. Doss, private communication.

${ }^{11}$ K. Stricker, H. McManus, and J. A. Carr, Phys. Rev. C 19 , 929 (1979).

${ }^{12}$ K. Nakai, T. Kobayashi, T. Numao, T. A. Shibata, J. Chiba, and K. Masutani, Phys. Rev. Lett. 44, 1446 (1980).

${ }^{13}$ K. Chen, Z. Fraenkel, G. Friedlander, J. R. Grover, J. M. Miller, and Y. Shimamoto, Phys. Rev. 166, 949 (1969).

${ }^{14}$ S. Nagamiya, M.-C. Lemaire, E. Moeller, S. Schnetzer, G. Shapiro, H. Steiner, and I. Tanihata, Phys. Rev. C 24, 971 (1981).

${ }^{15}$ D. R. F. Cochran, P. N. Dean, P. A. M. Gram, E. A. Knapp, E. R. Martin, D. E. Nagle, R. B. Perkins, W. J. Shlaer, H. A. Thiessen, and E. D. Theriot, Phys. Rev. D $\underline{6}, 3085$ (1972).

16D. S. Beder and P. Bendix, Nucl. Phys. $\underline{B} 26,597$ (1971).

${ }^{17}$ M. M. Sternheim and R. R. Silbar, Phys. Rev. D $\underline{6}$, 3117 (1972).

${ }^{18}$ R. R. Silbar and M. M. Sternheim, Phys. Rev. C $\underline{8}, 492$ (1973).

19J. F. Crawford, M. Daum, G. H. Eaton, R. Frosch, H. H. Hirschmann, R. Horisberger, J. W. McCulloch, E. Steiner, R. Hausamann, R. Hess, and D. Werren, Schweizerisches Institut fur Nuklearforschung Report 79-010, 1979.
${ }^{20}$ A. G. Meshkovskii, Iu. S. Pligin, Ia. Ia. Shalamov, and V. A. Shevanov, Zh. Eksp. Teor. Fiz. 1ㅣ, 987 (1956) [Sov. Phys.-JETP 4 , 842 (1957)].

${ }^{21}$ A. H. Rosenfeld, Phys. Rev. 96, 139 (1954).

${ }^{22}$ M. M. Block, S. Passman, and W. W. Havens, Jr., Phys. Rev. 88, 1239 (1952).

${ }^{23}$ A. H. Rosenfeld, Phys. Rev. 96, 130 (1954).

${ }^{24}$ E. Lillethun, Phys. Rev. 125, 665 (1962).

25J. Merritt and D. A. Hamlin, Phys. Rev. 99, 1523 (1955).

${ }^{26}$ Margaret Sandel, J. P. Vary, and S. I. A. Garpman, Phys. Rev. C 20, 744 (1979).

${ }^{27}$ L. C. L. Yuan and S. J. Lindenbaum, Phys. Rev. 103, 404 (1956).

${ }^{28}$ D. V. Bugg, A. J. Oxley, J. A. Zoll, J. G. Rushbrooke, V. E. Barnes, J. B. Kinson, W. P. Dodd, G. A. Doran, and L. Riddiford, Phys. Rev. 133, B1017 (1964).

${ }^{29}$ K. O. Oganesyan, Zh. Eksp. Teor. Fiz. 노, 1273 (1968) [Sov. Phys.-JETP 27, 679 (1968)].

30J. Chiba, K. Nakai, I. Tanihata, S. Nagamiya, H. Bowman, J. Ingersoll, and J. O. Rasmussen, Phys. Rev. C 20, 1332 (1979).

${ }^{31}$ K. Nakai, J. Chiba, I. Tanihata, M. Sasao, H. Bowman, S. Nagamiya, and J. O. Rasmussen, Phys. Rev. C $\underline{20}$, 2210 (1979).

${ }^{32}$ G. D. Harp, Phys. Rev. C $\underline{10}$, 2387 (1974).

${ }^{33} \mathrm{Y}$. Yariv and Z. Fraenkel, private communication.

${ }^{34}$ K. G. Libbrecht and S. E. Koonin, Phys. Rev. Lett. $\underline{43}$, 1581 (1979).

${ }^{35}$ R. Stock, Gesellschaft für Schwerionenforschung, Darmstadt, Report No. GSI-P-5-78, 1978 (unpublished), p. 66 .

${ }^{36}$ K. L. Wolf, Proceedings of the Workshop on Future Relativistic Heavy Ion Experiments, GSI Darmstadt 1980, Report No. GSI 81-6, 1981 (unpublished), p. 23.

${ }^{37}$ H. Späth, Comput. Rev. 4, 225 (1969).

38J. Knoll and J. Randrup, Nucl. Phys, A324, 445 (1979).

39J. Cugnon, D. Kinet, and J. Vandermuelen, Université de Liège report, 1981; J. Cugnon, Phys. Rev. C 22, 1885 (1980).

${ }^{40}$ W. Benenson, G. Bertsch, G. M. Crawley, E. Kashy, J. A. Nolen, Jr., H. Bowman, J. G. Ingersoll, J. O. Rasmussen, J. Sullivan, M. Koike, M. Sasao, J. Peter, and T. E. Ward, Phys. Rev. Lett. 43, 683 (1979); 44 , 54(E) (1980).

${ }^{41} \mathrm{~J}$. Cugnon and S. E. Koonin, Proceedings of the Workshop on Nuclear Dynamics, 1980, Granlibakken, Tahoe City, California, Lawrence Berkeley Laboratory Report LBL-10688, 1980.

${ }^{42}$ M. Gyulassy and S. K. Kauffman, Nucl. Phys. A362, 503 (1981).

${ }^{43}$ Y. Boneh, Z. Fraenkel, and I. Nebenzahl, Phys. Rev. 156,1305 (1967).

${ }^{44}$ K. A. Frankel, J. A. Bistirlich, R. Bossingham, H. R. Bowman, K. M. Crowe, C. J. Martoff, D. Murphy, J. O. Rasmussen, J. P. Sullivan, W. A. Zajc, J. P. Miller, 
O. Hashimoto, M. Koike, J. Péter, W. Benenson, G. M. Crawley, E. Kashy, J. A. Nolen, Jr., and J. Quebert (unpublished).

45J. Zimanyi, G. Fai, and B. Jakobsson, Phys. Rev. Lett. 43, 1705 (1979).
46J. Boguta, Phys. Lett. 109B, 251 (1982).

${ }^{47}$ J. O. Rasmussen, R. Frankel, J. Sullivan, D. Murphy, H. Bowman, K. Crowe, W. Zajc, H. M. A. Radi, and W. Benenson, Bull. Am. Phys. Soc. 27, 26 (1982). 